\title{
MixMo: Mixing Multiple Inputs for Multiple Outputs via Deep Subnetworks
}

\author{
Alexandre Ramé ${ }^{* \dagger 1}$, Rémy Sun*1,2 and Matthieu Cord ${ }^{1,3}$ \\ ${ }^{1}$ Sorbonne Université, CNRS, LIP6, Paris, France \\ ${ }^{2}$ Optronics \& Missile Electronics, Land \& Air Systems, Thales \\ ${ }^{3}$ Valeo.ai
}

\begin{abstract}
Recent strategies achieved ensembling "for free" by fitting concurrently diverse subnetworks inside a single base network. The main idea during training is that each subnetwork learns to classify only one of the multiple inputs simultaneously provided. However, the question of how to best mix these multiple inputs has not been studied so far.

In this paper, we introduce MixMo, a new generalized framework for learning multi-input multi-output deep subnetworks. Our key motivation is to replace the suboptimal summing operation hidden in previous approaches by a more appropriate mixing mechanism. For that purpose, we draw inspiration from successful mixed sample data augmentations. We show that binary mixing in features - particularly with rectangular patches from CutMix - enhances results by making subnetworks stronger and more diverse.

We improve state of the art for image classification on CIFAR-100 and Tiny ImageNet datasets. Our easy to implement models notably outperform data augmented deep ensembles, without the inference and memory overheads. As we operate in features and simply better leverage the expressiveness of large networks, we open a new line of research complementary to previous works. [
\end{abstract}

\section{Introduction}

Convolutional Neural Networks (CNNs) have shown exceptional performance in computer vision tasks, notably classification [42]. However, among other limitations, obtaining reliable predictions remains challenging [34, 58]. For additional robustness in real-world scenarios or to win Kaggle competitions, CNNs usually pair up with two practical strategies: data augmentation and ensembling.

\footnotetext{
${ }^{*}$ Equal contribution.

${ }^{\dagger}$ Correspondence to alexandre.rame@lip6.fr

Proceedings of IEEE International Conference on Computer Vision (ICCV), 2021
}

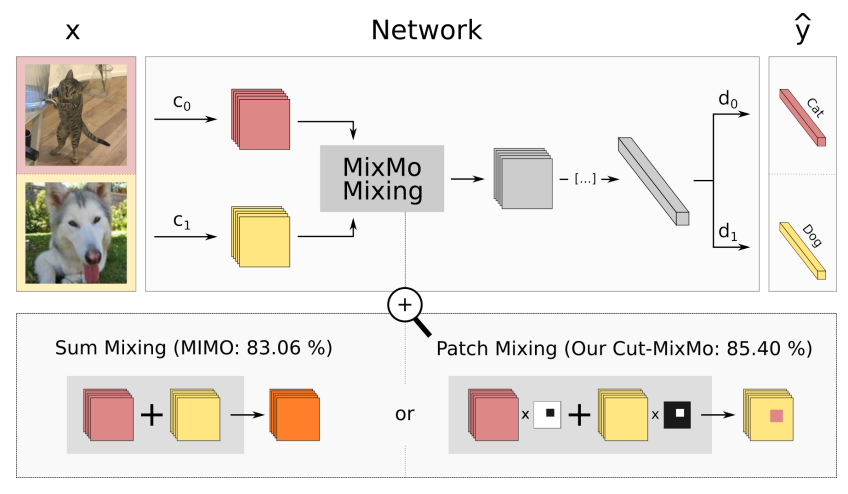

Figure 1: MixMo overview. We embed $M=2$ inputs into a shared space with convolutional layers $\left(c_{1}, c_{2}\right)$, mix them, pass the embedding through further layers and output 2 predictions via dense layers $\left(d_{1}, d_{2}\right)$. The key point of our MixMo is the mixing block. Mixing with patches performs better than basic summing: $85.40 \%$ vs. $83.06 \%$ (MIMO [30]) on CIFAR-100 with WRN-28-10.

Data augmentation reduces overfitting and improves generalization, notably by diversifying training samples [51]. Traditional approaches are label-preserving. In contrast, recent mixed sample data augmentation (MSDA) create artificial samples by mixing multiple inputs and their labels proportionally to a ratio $\lambda$. The seminal work Mixup [86] linearly interpolates pixels while Manifold Mixup [76] interpolates latent features in the network. Binary masking MSDAs [21, 29, 41] such as CutMix [83] have since diversified mixed samples by pasting patches from one image onto another in place of interpolation.

Aggregating predictions from a diverse set of neural networks (i.e. with different failure cases) strongly improves generalization [14, 28, 43], notably uncertainty estimation [2, 27, 58]. An ensemble of several small networks usually performs better than one large network empirically [9, 50]. Yet, unfortunately, ensembling is costly in time and memory 
both at training and inference: this often limits applicability.

In this paper, we propose MixMo, a new generalized multi-input multi-output framework: we train a base network with $M \geq 2$ inputs and outputs. This way, we fit $M$ independent subnetworks [23, 30, 66] defined by an input/output pair and a subset of network weights. This is possible as large networks only leverage a subset of their weights [19]. Rather than pruning (ie, eliminating) inactive filters [44, 47], we seek to fully use the available neurons and over parameterization through multiple subnetworks.

The challenge is to prevent homogenization and enforce diversity among subnetworks with no structural differences. Thus, we consider $M$ (input, label) pairs at the same time in training: $\left\{\left(x_{i}, y_{i}\right)\right\}_{0 \leq i<M} . M$ images are treated simultaneously, as shown on Fig. 1 with $M=2$. The $M$ inputs are encoded by $M$ separate convolutional layers $\left\{c_{i}\right\}_{0 \leq i<M}$ into a shared latent space before being mixed. The representation is then fed to the core network, which finally branches out into $M$ dense layers $\left\{d_{i}\right\}_{0 \leq i<M}$. Diverse subnetworks naturally emerge as $d_{i}$ learns to classify $y_{i}$ from input $x_{i}$. At inference, the same image is repeated $M$ times: we obtain ensembling "for free" by averaging $M$ predictions.

The key divergent point between MixMo variants lies in the multi-input mixing block that seeks features independence. Should the merging be a basic summation or a concatenation, we would recover MIMO [30] or respectively Aggregated Learning [66] - which both featured this multiinput multi-output strategy.

Our main intuition is simple: we see summing as a balanced and restrictive form of Mixup [86] where $\lambda=\frac{1}{M}$. By analogy, we draw from the considerable MSDA literature to design a more appropriate mixing block. In particular, we leverage binary masking methods to ensure subnetworks diversity. Our framework allows us to create a new Cut-MixMo variant inspired by CutMix [83], and illustrated in Fig. 1: a patch of features from the first input is pasted into the features from the second input.

This asymmetrical mixing also raises new questions regarding information flow in the network's features. We tackle the imbalance between the multiple classification training tasks via a new weighting scheme. Conversely, MixMo's double nature as a new mixing augmentation in features yields important insights on traditional MSDA.

In summary, our contributions are threefold:

1. We propose a general framework, MixMo, connecting two successful fields: mixing samples data augmentations \& multi-input multi-output ensembling.

2. We identify the appropriate mixing block to best tackle the diversity/individual accuracy trade-off in subnetworks: our easy to implement Cut-MixMo benefits from the synergy between CutMix and ensembling.

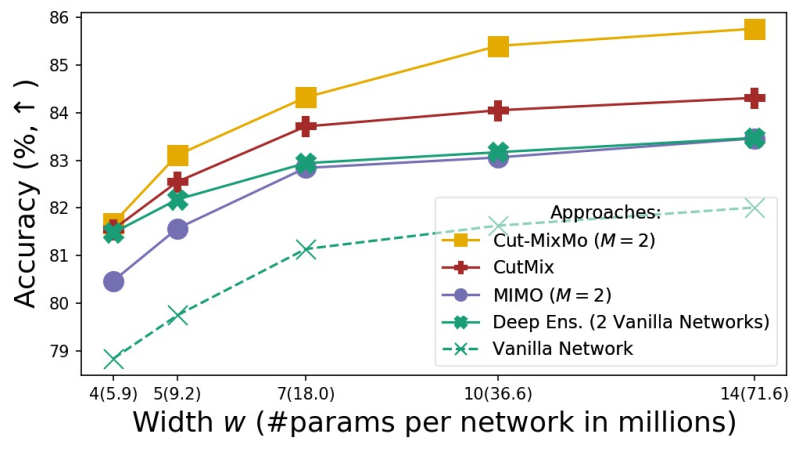

Figure 2: Main results. CIFAR-100 with WRN-28-w. Our Cut-MixMo variant (patch mixing and $M=2$ ) surpasses CutMix and deep ensembles (with half the parameters) by leveraging over-parameterization in wide networks.

3. We design a new weighting of the loss components to properly leverage the asymmetrical inputs mixing.

We demonstrate excellent accuracy and uncertainty estimation with MixMo on CIFAR-10/100 and Tiny ImageNet. Specifically, Cut-MixMo with $M=2$ reaches state of the art on these standard datasets: as exhibited by Fig. 22 it outperforms CutMix, MIMO and deep ensembles, at (almost) the same inference cost as a single network.

\section{Related work}

\subsection{Data augmentation}

CNNs are known to memorize the training data [85] and make overconfident predictions [25] to the detriment of generalization on new test examples. Data Augmentation (DA) inflates the training dataset's size by creating artificial samples from available labeled data. Beyond slight perturbations (e.g. rotation), recent works [11, 35] apply stronger transformations [33]. CutOut [13] randomly deletes regions of images in training and prevents models from focusing on a single pixels region, similarly to how regularizations like Dropout [67] or DropBlock [24] force networks to leverage multiple features.

Mixed Sample Data Augmentation (MSDA) recently expanded the notion of DA. From pairs of labeled samples $\left\{\left(x_{i}, y_{i}\right),\left(x_{k}, y_{k}\right)\right\}$, they create virtual samples: $\left(m_{x}\left(x_{i}, x_{k}, \lambda\right), \lambda y_{i}+(1-\lambda) y_{k}\right)$ where $\lambda \sim \operatorname{Beta}(\alpha, \alpha)$. [48] shows that mixing the targets differently than this linear interpolation may cause underfitting and unstable learning. Indeed, approaches mainly focus on developing the most effective input mixing $m_{x}$. In [38, 72, 73, 86], $m_{x}$ performs a simple linear interpolation between pixels: $e . g$ in Mixup [86], $m_{x}\left(x_{i}, x_{k}, \lambda\right)=\lambda x_{i}+(1-\lambda) x_{k}$. Theoretically, it regularizes outside the training distribution [5, 26, 87] and applies label smoothing [53, 61]. 
CutMix draws from Mixup and CutOut [13] by pasting a patch from $x_{k}$ onto $x_{i}: m_{x}\left(x_{i}, x_{k}, \lambda\right)=\mathbb{1}_{m} \odot x_{i}+$ $\left(\mathbb{1}-\mathbb{1}_{m}\right) \odot x_{k}$ where $\odot$ represents the element-wise product and $\mathbb{1}_{m}$ a binary mask with average value $\lambda$. CutMix randomly samples squares, which often leads to rectangular masks due to boundary effects. Such non-linear binary masking improves generalization [68, 70] by increasing dataset: it creates new images with usually disjoint patches [29]. [3, 17] seek more diverse transformations via arbitrarily shaped masks: proposals range from cow-spotted masks [21] to masks with irregular edges [29]. As masking of discriminative regions may cause label misallocation [26], [41, 74] try to alleviate this issue with costly saliency heatmaps [65]. Yet, ResizeMix [63] shows that they perform no better than random selection of patch locations.

In addition to Manifold Mixup [76], only a few works [17, 46, 81, 83] have tried to mix intermediate latent features as we do. Our goals and methods are however quite different, as shown later in Section 3.4. In brief, they mix deep features to smooth the decision boundaries, while we mix shallow features only so that inputs can remain distinct.

\subsection{Ensembling}

Like [79], we explore combining DA with another standard technique in machine learning: ensembling [14, 28]. For improved performances, aggregated members should be both accurate and diverse [57, 62, 64]. Deep ensembles [43] (DE) simultaneously train multiple networks with different random initializations converging towards different explanations for the training data [18, 80].

Ensembling's fundamental drawback is the inherent computational and memory overhead, which increases linearly with the number of members. This bottleneck is typically addressed by sacrificing either individual performance or diversity in a complex trade-off. Averaging predictions from several checkpoints on the training process, i.e. snapshot ensembles [37, 39], fails to explore multiple local optima [2, 18, 80]. So does Monte Carlo Dropout [22]. The recent BatchEnsemble [16] is parameter-efficient, yet requires multiple forward passes. TreeNets [45, 69] reduce training and inference cost by sharing low-level layers. MotherNets [78] share first training epochs between members. However, sharing reduces diversity.

Very recently, the multi-input multi-output MIMO [30] achieves ensemble almost "for free": all of the layers except the first convolutional and last dense layers are shared $(\approx+1 \%$ \#parameters). [66] motivated a related Aggregated Learning to learn concise representations with arguments from information bottleneck [71]. The idea is that overparameterized CNNs [19, 52, 60] can fit multiple subnetworks [75]. The question is how to prevent homogenization among the simultaneously trained subnetworks. Facing a similar challenge, [23] includes stochastic channel recombi- nation; [15] relies on predefined binary masks; in GradAug [82], subnetworks only leverage the first channels up to a given percentage. In contrast, MIMO does not need structural differences among subnetworks: they learn to build their own paths while being as diverse as in DE.

\section{MixMo framework}

We first introduce the main components of our MixMo strategy, summarized in Fig. 3 we mix multiple inputs to obtain multiple outputs via subnetworks. We highlight the key mixing block combining information from inputs, and our training loss based on a dedicated weighting scheme.

We mainly study $M=2$ subnetworks here, both for clarity and as it empirically performs best in standard parameterization regimes. For completeness, we straightforwardly generalize to $M>2$ in Section 3.5

\subsection{General overview}

We leverage a training classification dataset $D$ of i.i.d. pairs of associated image/label $\left\{x_{i}, y_{i}\right\}_{i=1}^{|D|}$. We randomly sample a subset of $|B|$ samples $\left\{x_{i}, y_{i}\right\}_{i \in B}$ that we randomly shuffle via permutation $\pi$. Our training batch is $\left\{\left(x_{i}, x_{j}\right),\left(y_{i}, y_{j}\right)\right\}_{i \in B, j=\pi(i)}$. The loss $\mathcal{L}_{\text {MixMo }}$ is averaged over these $|B|$ samples: the networks' weights are updated through backpropagation and gradient descent.

Let's focus on the training sample $\left\{\left(x_{0}, x_{1}\right),\left(y_{0}, y_{1}\right)\right\}$. In MixMo, both inputs are separately encoded (see Fig. 1) into the shared latent space with two different convolutional layers (with 3 input channels each and no bias term): $x_{0}$ via $c_{0}$ and $x_{1}$ via $c_{1}$. To recover a strictly equivalent formulation to MIMO [30], we simply sum the two encodings: $c_{0}\left(x_{0}\right)+c_{1}\left(x_{1}\right)$. Indeed, MIMO merges inputs through channel-wise concatenation in pixels: MIMO's first convolutional layer (with 6 input channels and no bias term) hides the summing operation in the output channels.

Explicitly highlighting the underlying mixing leads us to consider a generalized multi-input mixing block $\mathcal{M}$. This manifold mixing presents a unique opportunity to tackle the ensemble diversity/individual accuracy trade-off and to improve overall ensemble results (see Section 3.2). The shared representation $\mathcal{M}\left(c_{0}\left(x_{0}\right), c_{1}\left(x_{1}\right)\right)$ feeds the next convolutional layers. We note $\kappa$ the mixing ratio between inputs.

The core network $\mathcal{C}$ handles features that represent both inputs simultaneously. The dense layer $d_{0}$ predicts $\hat{y}_{0}=$ $d_{0}\left[\mathcal{C}\left(\mathcal{M}\left\{c_{0}\left(x_{0}\right), c_{1}\left(x_{1}\right)\right\}\right)\right]$ and targets $y_{0}$, while $d_{1}$ targets $y_{1}$. Thus, the training loss is the sum of two cross-entropies $\mathcal{L}_{\mathrm{CE}}$ weighted by parametrized function $w_{r}$ (defined in Section 3.3 to balance the asymmetry when $\kappa \neq 0.5$ :

$$
\mathcal{L}_{\text {MixMo }}=w_{r}(\kappa) \mathcal{L}_{\mathrm{CE}}\left(y_{0}, \hat{y}_{0}\right)+w_{r}(1-\kappa) \mathcal{L}_{\mathrm{CE}}\left(y_{1}, \hat{y}_{1}\right) .
$$

At inference, the same input $x$ is repeated twice: the core network $\mathcal{C}$ is fed the sum $c_{0}(x)+c_{1}(x)$ that preserves maximum information from both encodings. Then, the diverse 
predictions are averaged: $\frac{1}{2}\left(\hat{y}_{0}+\hat{y}_{1}\right)$. This allows us to benefit from ensembling in a single forward pass.

\subsection{Mixing block $\mathcal{M}$}

The mixing block $\mathcal{M}$ - which combines both inputs into a shared representation - is the cornerstone of MixMo. Our main intuition was to analyze MIMO as a simplified Mixup variant where the mixing ratio $\kappa$ is fixed to 0.5 . MixMo generalized framework encompasses a wider range of variants inspired by MSDA mixing methods. Our first main variant - Linear-MixMo - fully extends Mixup. The mixing block is $\mathcal{M}_{\text {Linear-MixMo }}\left(l_{0}, l_{1}\right)=2\left[\kappa l_{0}+(1-\kappa) l_{1}\right]$, where $l_{0}=c_{0}\left(x_{0}\right), l_{1}=c_{1}\left(x_{1}\right)$ and $\kappa \sim \operatorname{Beta}(\alpha, \alpha)$ with $\alpha$ the concentration parameter. The second and more effective variant Cut-MixMo adapts the patch mixing from CutMix:

$$
\mathcal{M}_{\text {Cut-MixMo }}\left(l_{0}, l_{1}\right)=2\left[\mathbb{1}_{\mathcal{M}} \odot l_{0}+\left(\mathbb{1}-\mathbb{1}_{\mathcal{M}}\right) \odot l_{1}\right],
$$

where $\mathbb{1}_{\mathcal{M}}$ is a binary mask with area ratio $\kappa \sim \operatorname{Beta}(\alpha, \alpha)$, valued at 1 either on a rectangle or on the complementary of a rectangle. In brief, a patch from $c_{0}\left(x_{0}\right)$ is pasted onto $c_{1}\left(x_{1}\right)$, or vice versa. This binary mixing in Cut-MixMo advantageously replaces the linear interpolation in MIMO and Linear-MixMo: subnetworks are more accurate and more diverse, as shown empirically in Fig.7.

First, binary mixing in $\mathcal{M}$ trains stronger individual subnetworks for the same reasons why CutMix improves over Mixup. In a nutshell, linear MSDAs [76, 86] produce noisy samples [5] that lead to robust representations. As MixMo tends to distribute different inputs on nonoverlapping channels (as discussed later in Fig. 4a), this regularization hardly takes place anymore in $\mathcal{M}_{\text {Linear-MixMo }}$. On the contrary, by masking features, we simulate common object occlusion problems. This spreads subnetworks' focus across different locations: the two classifiers are forced to find information relevant to their assigned input at disjoint locations. This occlusion remains effective as the receptive field in this first shallow latent space remains small.

Secondly, linear interpolation is fundamentally ill-suited to induce diversity as full information is preserved from both inputs. CutMix on the other hand explicitly increases dataset diversity by presenting patches of images that do not normally appear together. Such benefits can be directly transposed to $\mathcal{M}_{\text {Cut-MixMo }}$ : binary mixing with patches increases randomness and diversity between the subnetworks. Indeed, in a similar spirit to bagging [4], different samples are given to the subnetworks. By deleting asymmetrical complementary locations from the two inputs, subnetworks will not rely on the same region and information. Overall, they are less likely to collapse on close solutions.

\subsection{Loss weighting $w_{r}$}

Asymmetries in the mixing mechanism can cause one input to overshadow the other. Notably when $\kappa \neq 0.5$,

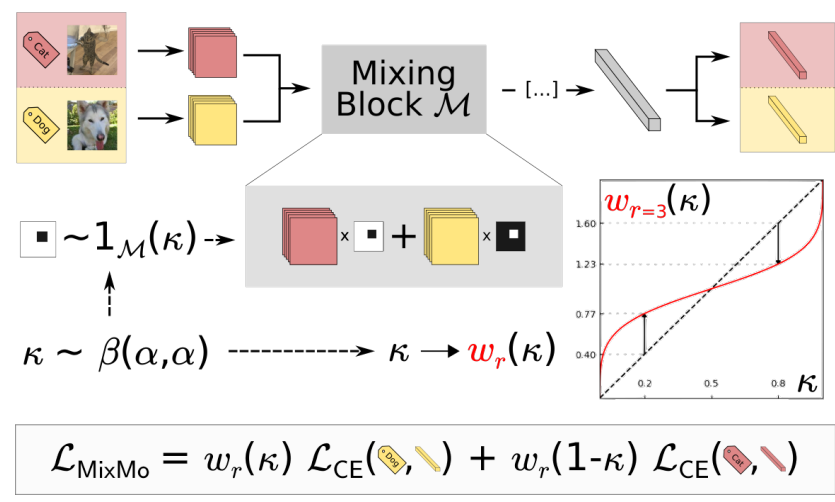

Figure 3: Cut-MixMo training. We sample a mixing mask given $\kappa$, and balance the losses with $w_{r}(\kappa)$ from Eq. 3 .

the predominant input may be easier to predict. We seek a weighting function $w_{r}$ to balance the relative importance of the two $\mathcal{L}_{\mathrm{CE}}$ in $\mathcal{L}_{\text {MixMo. }}$ This weighting modifies the effective learning rate, how gradients flow in the network and overall how mixed information is represented in features. In this paper, we propose to weight via the parametrized:

$$
w_{r}(\kappa)=2 \frac{\kappa^{1 / r}}{\kappa^{1 / r}+(1-\kappa)^{1 / r}} .
$$

This defines a family of functions indexed by the parameter $r$, visualized for $r=3$ in red on Fig. 3. See Appendix 6.1 for complementary visualizations. This power law provides a natural relaxation between two extreme configurations. The first extreme, $r=1, w_{1}(\kappa)=2 \kappa$, is in line with linear label interpolation in MSDA. The resulting imbalance in each subnetwork's contribution to $\mathcal{L}_{\text {MixMo }}$ causes lopsided updates. While it promotes diversity, it also reduces regularization: the overshadowed input has a reduced impact on the loss. The opposite extreme, $r \rightarrow \infty, w_{\infty}(\kappa) \rightarrow 1$, removes reweighting. Consequently, $w_{r}$ inflates the importance of hard under-represented inputs, à la Focal Loss [49]. However, minimizing the role of the predominant inputs destabilizes training. Overall, we empirically observe that moderate values of $r$ perform best as they trade off pros and cons from both extremes.

Interestingly, the proper weighting of loss components is also a central theme in multi-task learning [6, 8]. While it aims at predicting several tasks from a shared input, MixMo predicts a shared task from several different inputs. Beyond this inverted structure, we have similar issues: e.g. gradients for one task can be detrimental to another conflicting task. Fortunately, MixMo presents an advantage: the exact ratios $\kappa$ and $1-\kappa$ of each task are known exactly.

\subsection{From manifold mixing to MixMo}

We have discussed at length how we extend multi-input multi-output frameworks by borrowing mixing protocols 


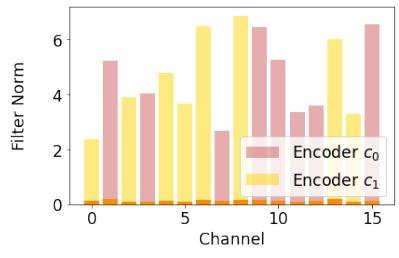

(a) Filters $l_{1}$-norms of the input encoders $c_{0}$ and $c_{1}$.

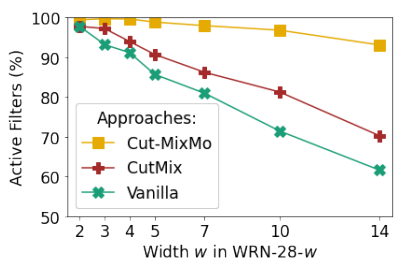

(b) Proportion of active filters in the core network vs. width $w$.
Figure 4: Influence of MixMo on network utilization. (a) The encoders have separate channels: the two subsequent classifiers can differentiate the two inputs. (b) Less filters are strongly active $\left(\left\|f_{i}\right\|_{1} \geq 0.4 \times \max _{f \in \text { layer }}\|f\|_{1}\right)$ in wider networks: Cut-MixMo reduces this negative point.

from MSDA. Now we reversely point out how our MixMo diverges from MSDA schemes. At first glimpse, the idea is the same as manifold mixing [17, 46, 76]: $M=2$ inputs are encoded into a latent space to be mixed before being fed to the rest of the network. Yet, while they mix at varying depths, we only mix in the shallowest space. Specifically, we only mix in features - and not in pixels - to allow separate encodings of the inputs: they need to remain distinct in the mixed representation for the subsequent classifiers.

Hence our two key differences: first, MixMo uses two separated encoders (one for each input), and second, it outputs two predictions instead of a single one. Indeed, MSDAs use a single classifier that targets a unique soft label reflecting the different classes via linear interpolation. MixMo instead chooses to fully leverage the composite nature of mixed samples and trains separated dense layers, $d_{0}$ and $d_{1}$, ensembled "for free" at test time.

Section 4.3.5 demonstrates that MixMo works because it also uses two different encoders $c_{0}$ and $c_{1}$. While training two classifiers may seem straightforward in MSDA, it actually raises a troubling question: which input should each classifier predicts ? Having two encoders provides a simple solution: the network is divided in two subnetworks, one for each input. Their separability is easily observed: Fig. 4a shows the $l_{1}$-norm of the 16 filters for the two encoders (WRN-28-10 on CIFAR-100). Each filter norm is far from zero in only one of the two encoders: $c_{0}\left(x_{0}\right)$ and $c_{1}\left(x_{1}\right)$ separate the inputs in different dimensions which allows subsequent layers to treat them differently.

This leads MixMo to use most available filters. Following the structured pruning literature [47], we consider in Fig. $4 \mathrm{~b}$ that a filter (in a layer of the core network) is active if its $l_{1}$-norm is at least $40 \%$ of the $l_{1}$-norm from its layer's most active filter (see Appendix 6.2. This illustrates the known increase in sparsity in wider networks. Conversely, having 2 subnetworks in MixMo enables the weights ignored by one subnetwork to be leveraged by the other.

\subsection{Generalization to $M \geq 2$ subnetworks}

Most of the framework is easily extended by optimizing $\mathcal{L}_{\text {MixMo }}=\sum_{0 \leq i<M} M \frac{\kappa_{i}^{1 / r}}{\sum_{j} \kappa_{j}^{1 / r}} \mathcal{L}_{\mathrm{CE}}\left(y_{i}, \hat{y}_{i}\right)$ with $\left\{\kappa_{i}\right\} \sim$ $\operatorname{Dir}(\alpha)$ from a Dirichlet distribution (see Appendix 6.3 . The key change is that $\mathcal{M}$ now needs to handle more than 2 inputs: $\left\{c_{i}\left(x_{i}\right)\right\}_{0 \leq i<M}$. While linear interpolation is easily generalized, Cut-MixMo has several possible extensions: in our experiments, we first linearly interpolate between $M-1$ inputs and then patch in a region from the $M$-th.

\section{Experiments}

We evaluate MixMo efficiency on standard image classification datasets: CIFAR- $\{10,100\}$ [42] and Tiny ImageNet [10]. We equally track accuracies $(\operatorname{Top}\{1,5\}, \uparrow)$ and the calibrated Negative Log-Likelihood $\left(\mathrm{NLL}_{c}, \downarrow\right)$. Indeed, [2] shows that we should compare in-domain uncertainty estimations after temperature scaling (TS) [25]: we thus split the test set in two and calibrate (after averaging in ensembles) with the temperature optimized on the other half, as in [50, 64]. We nonetheless report NLL (without TS) along with the Expected Calibration Error [54] in Appendix 6.5

\subsection{Implementation details}

We mostly study the Linear-MixMo and Cut-MixMo variants with $M=2$. We set hyper-parameter $r=3$ (see Section 4.3.3) $\alpha=2$ performs better than 1 (see Appendix 6.8. In contrast, MIMO [30] refers to linear summing, like Linear-MixMo, but with $\kappa=0.5$ instead of $\kappa \sim \operatorname{Beta}(\alpha, \alpha)$.

Different mixing methods create a strong train-test distribution gap [5, 51]. Thus, in Cut-MixMo we actually substitute $\mathcal{M}_{\text {Cut-MixMo }}$ for $\mathcal{M}_{\text {Linear-MixMo with probability }}$ $1-p$ to accommodate for the summing in $\mathcal{M}$ at inference. We set the probability of patch mixing during training to $p=0.5$, with linear descent to 0 over the last twelfth of training epochs (see pseudocode 1 in Appendix).

When MixMo is combined with CutMix, the pixels in puts are: $\left(m_{x}\left(x_{i}, x_{k}, \lambda\right), m_{x}\left(x_{j}, x_{k^{\prime}}, \lambda^{\prime}\right)\right)$ with interpolated targets $\left.\left(\lambda y_{i}+(1-\lambda) y_{k}, \lambda^{\prime} y_{j}+\left(1-\lambda^{\prime}\right) y_{k^{\prime}}\right)\right)$, where $k, k^{\prime}$ are randomly sampled and $\lambda, \lambda^{\prime} \sim \operatorname{Beta}(1,1)$.

MIMO duplicates samples $b$ times via batch repetition: $x_{i}$ will be associated with $x_{\pi(i)}$ and $x_{\pi^{\prime}(i)}$ in the same batch if $b=2$. As the batch size remains fixed, the count of unique samples per batch and the learning rate is divided by $b$. Conversely, the number of steps is multiplied by $b$. Overall, this stabilizes training but multiplies its cost by $b$. We thus indicate an estimated (training/inference) overhead (wrt. vanilla training) in the time column of our tables. Note that some concurrent approaches also lengthen training: e.g. GradAug $[82]$ via multiple subnetworks predictions $(\approx \times 3)$.

We provide more details in Appendix 6.4 and will open source our PyTorch [59] implementation. 
Table 1: Main results: WRN-28-10 on CIFAR. Bold highlights best scores, $\dagger$ marks approaches not re-implemented.

\begin{tabular}{|c|c|c|c|c|c|c|}
\hline \multicolumn{2}{|l|}{ Dataset } & \multicolumn{3}{|c|}{ CIFAR-100 } & \multicolumn{2}{|c|}{ CIFAR-10 } \\
\hline Approach & $\begin{array}{c}\text { Time } \\
\text { Tr./Inf. }\end{array}$ & $\begin{array}{l}\text { Top1 } \\
\%, \uparrow \\
\end{array}$ & $\begin{array}{l}\text { Top5 } \\
\%, \uparrow \\
\end{array}$ & $\begin{array}{c}\text { NLL }_{c} \\
10^{-2}, \downarrow\end{array}$ & $\begin{array}{l}\text { Top1 } \\
\%, \uparrow \\
\end{array}$ & $\begin{array}{c}\mathrm{NLL}_{c} \\
10^{-2}, \downarrow\end{array}$ \\
\hline Vanilla & \multirow{5}{*}{$1 / 1$} & 81.63 & 95.49 & 73.9 & 96.34 & 12.6 \\
\hline Mixup & & 83.44 & 95.92 & 65.7 & 97.07 & 11.2 \\
\hline Manifold Mixup ${ }^{\dagger}$ & & 81.96 & 95.51 & 73.4 & 97.45 & 12.2 \\
\hline CutMix & & 84.05 & 96.09 & 64.8 & 97.23 & 9.9 \\
\hline $\operatorname{ResizeMix}^{\dagger}$ & & 84.31 & - & - & 97.60 & - \\
\hline Puzzle-Mix ${ }^{\dagger}$ & $2 / 1$ & 84.31 & 96.46 & 66.8 & - & - \\
\hline $\operatorname{GradAug}^{\dagger}$ & \multirow{2}{*}{$3 / 1$} & 84.14 & 96.43 & - & - & - \\
\hline$+\mathrm{CutMix}^{\dagger}$ & & 85.51 & 96.86 & - & - & - \\
\hline Mixup BA ${ }^{\dagger}$ & $7 / 1$ & 84.30 & - & - & 97.80 & - \\
\hline DE (2 Nets) & \multirow{2}{*}{$2 / 2$} & 83.17 & 96.37 & 66.4 & 96.67 & 11.1 \\
\hline + CutMix & & 85.74 & 96.82 & 57.1 & 97.52 & 8.6 \\
\hline MIMO & \multirow{5}{*}{$2 / 1$} & 82.40 & 95.78 & 68.8 & 96.38 & 12.1 \\
\hline Linear-MixMo & & 82.54 & 95.99 & 67.6 & 96.56 & 11.4 \\
\hline + CutMix & & 84.69 & 97.12 & 57.2 & 97.32 & 9.4 \\
\hline Cut-MixMo & & 84.38 & 96.94 & 56.3 & 97.31 & 8.9 \\
\hline + CutMix & & 85.18 & 97.20 & 54.5 & 97.45 & 8.4 \\
\hline MIMO & \multirow{5}{*}{$4 / 1$} & 83.06 & 96.23 & 66.1 & 96.74 & 11.4 \\
\hline Linear-MixMo & & 83.08 & 96.26 & 65.6 & 96.91 & 10.8 \\
\hline + CutMix & & 85.47 & 97.04 & 55.8 & 97.68 & 8.7 \\
\hline Cut-MixMo & & 85.40 & 97.22 & 53.5 & 97.51 & 8.1 \\
\hline + CutMix & & 85.77 & 97.42 & 52.4 & 97.73 & 7.9 \\
\hline
\end{tabular}

\subsection{Main results on CIFAR-100 and CIFAR-10}

Tab. 1 reports averaged scores over 3 runs for our main experiment on CIFAR with WRN-28-10 [84]. We re-use the hyper-parameters given in MIMO [30]. Cut-MixMo reaches $\left(85.40 \%\right.$ Top1, $0.535 \mathrm{NLL}_{c}$ ) on CIFAR-100 with $b=4$ : it surpasses our Linear-MixMo $(83.08 \%, 0.656)$ and MIMO $(83.06 \%, 0.661)$. Cut-MixMo sets a new state of the art when combined with CutMix $(85.77 \%, 0.524)$. Results remain strong when $b=2$ : Cut-MixMo $(84.38 \%, 0.563)$ proves better on its own than traditional DE [43], and MSDAs like MixUps [86, 76] or the stronger CutMix variant [83]. On CIFAR-10, we see similar trends: Cut-MixMo reaches 0.081 in $\mathrm{NLL}_{c}, 0.079$ with CutMix. Yet, the costlier batch augmented Mixup BA [36] edges it out in Top1.

Fig. 5 shows how MixMo grows stronger than DE (green curves) as width $w$ in WRN-28- $w$ increases. The parameterization becomes appropriate at $w=4$ : Cut-MixMo (yellow curves) then matches DE - with half the parameters in Fig. 5a and its subnetworks match a vanilla network in Fig. 5b. Beyond, MixMo better uses over-parameterization: Cut-MixMo+CutMix surpasses DE+CutMix in $\mathrm{NLL}_{c}$ for $w \geq 5$, and this is true in Top1 for $w \geq 10$. Compared to our strong Linear-MixMo+CutMix (purple curves), CutMixMo performs similarly in Top1, and better with CutMix for $w \geq 4$. While Linear-MixMo and DE learn from occlusion, Cut-MixMo also benefits from CutMix, notably from the induced label smoothing. Overall, Cut-MixMo, even without CutMix, significantly better estimates uncertainty.

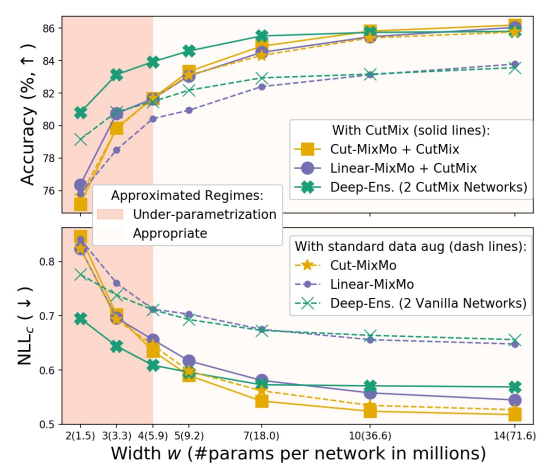

(a) Ensemble Top1 and $\mathrm{NLL}_{c}$.

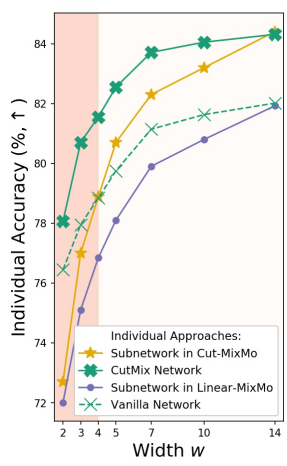

(b) Individual Top1.
Figure 5: Parameters efficiency (metrics/\#params). CIFAR-100 with WRN-28- $w, b=4$. Comparisons between (a) ensemble and some of their (b) individual counterparts.

\subsection{MixMo analysis on CIFAR-100 w/ WRN-28-10}

\subsubsection{Training time}

We have just seen that CutMix improves Linear-MixMo at varying widths $w$, but not enough to match Cut-MixMo in $\mathrm{NLL}_{c}$ : CutMix can not fully compensate for the advantages from patch mixing over linear interpolation. We recover this finding in Fig. 6 this time at varying batch repetition $b \in$ $\{1,2,4\}$ when $w=10$. Moreover, Cut-MixMo outperforms $\mathrm{DE}$ for the same training time. Indeed, MixMo variants trained with a given $b$ matches the training time of DE with $N=b$ networks. In the rest of this section, we set $b=2$.

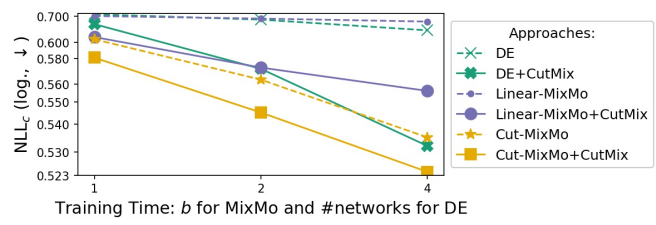

Figure 6: $\mathbf{N L L}_{c}(\downarrow)$ improves with longer training, via batch repetitions (MixMo) or additional networks (DE).

\subsubsection{The mixing block $\mathcal{M}$}

Tab. 2 compares performance for several mixing blocks [17, 29, 68, 83]. No matter the shape (illustrated in Appendix 6.7), binary masks perform better than linear mixing: the cow-spotted mask $(84.17 \%, 0.561)$ [20, 21] notably performs well. The basic CutMix patching $(84.38 \%, 0.563)$ is nevertheless more accurate and was our main focus.

Table 2: $\mathcal{M}$ inspired by various MSDA approaches.

\begin{tabular}{c|c|cccccc}
\hline $\mathcal{M}$ & Mixup & Horiz. & Vertical & PatchUp 2D & FMix & CowMask & CutMix \\
approach & {$[86]$} & Concat. & Concat. & {$[17]$} & {$[29]$} & {$[20,21]$} & {$[83]$} \\
\hline Top1 $\uparrow$ & 82.5 & 82.78 & 84.00 & 84.16 & 83.76 & 84.17 & $\mathbf{8 4 . 3 8}$ \\
NLL $_{c} \downarrow$ & 0.676 & 0.627 & 0.573 & 0.581 & 0.602 & $\mathbf{0 . 5 6 1}$ & 0.563 \\
\hline
\end{tabular}


We further study the impact of patch mixing through the lens of the ensemble diversity/individual accuracy trade off. As in [64], we measure diversity via the pairwise ratio-error [1] $\left(d_{r e}, \uparrow\right)$, defined as the ratio between the number of different errors and simultaneous errors for two predictors. In Fig. 7 and 8 , we average metrics over the last 10 epochs.

As argued in Section 3.2, patch mixing increases diversity compared to linear mixing in Fig. 7 As the probability $p$ of patch mixing grows, so does diversity: from $d_{r e}(p=0.0) \approx 0.78$ (Linear-MixMo) to $d_{r e}(p=0.5) \approx 0.85$ (Cut-MixMo). We provide associated training dynamics in Appendix 6.6 In contrast, DE has $d_{r e} \approx 0.76$ while MIMO has $d_{r e} \approx 0.77$ on the same setup. Increasing $p$ past 0.6 boosts diversity even more at the cost of subnetworks' accuracies: this is due to underfitting and an increased test-train distribution gap. $p \in[0.5,0.6]$ is thus the best trade off.

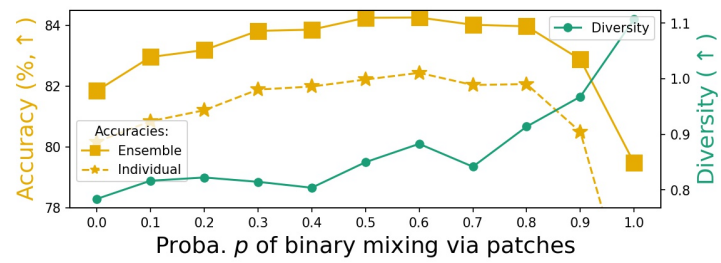

Figure 7: Diversity/accuracy as function of $p$ with $r=3$.

\subsubsection{Weighting function $w_{r}$}

We analyze the impact of the parameter $r$ in the reweighting function $w_{r}$. Higher values tend to remove reweighting, as shown in Appendix 6.1 they strongly decrease diversity in Fig. 8. The opposite extreme with $r=1$ increases diversity via lopsided gradient updates but it degrades accuracy. We speculate it under-emphasizes hard samples. The range $r \in$ $[3,6]$ strikes a good balance: results remain high and stable.

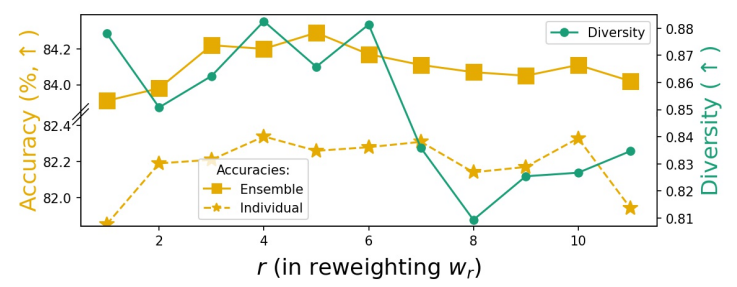

Figure 8: Diversity/accuracy as function of $r$ with $p=0.5$.

\subsubsection{Generalization to $M \geq 2$ subnetworks}

We try to generalize MixMo to more than $M=2$ subnetworks in Fig. 9. Cut-MixMo's subnetworks perform at $82.3 \%$ when $M=2$ vs. $79.5 \%$ when $M=3$. In MIMO, it's $79.8 \%$ vs. $77.7 \%$. Because subnetworks do not share features, higher $M$ degrades their results: only two can fit seamlessly. Ensemble Top1 overall decreases in spite of the additional predictions, as already noticed in MIMO [30].

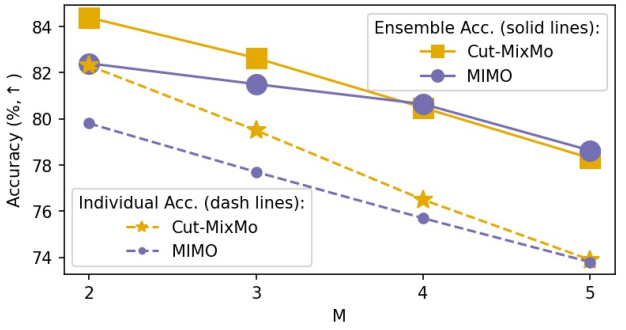

Figure 9: Ensemble/individual accuracies for $M \geq 2$.

This reflects MixMo's strength in over-parametrized regimes, but also its limitations with fewer parameters when subnetworks underfit (recall previous Fig. 55). Facing similar findings, MIMO [30] introduced input repetition so that subnetworks share their features, at the cost of drastically reducing diversity. Our generalization may be extended by future approaches whose mixing blocks (perhaps not inspired by MSDA) would tackle these issues.

\subsubsection{Multiple encoders and classifiers}

In Section 3.4. we compared MixMo and MSDA. Tab. 3 confirms the need for 2 encoders and 2 classifiers. With 1 classifier and linearly interpolated labels (in the same spirit as [7]), the 2 encoders perform worse than 1 encoder. With 1 shared encoder and 2 classifiers, it is not clear which input each classifier should target. In the first naive $\ominus$, we randomly associate the 2 classifiers and the 2 inputs (encoded with the same encoder). This $\ominus$ variant yields poor results. In $\otimes$, the first classifier tries to predict the label from the predominant input, the second targets the other input: $\otimes$ reaches 0.598 vs. 0.563 for Cut-MixMo.

\subsection{Robustness to image corruptions}

Deep networks' results decrease when facing unfamiliar samples. To measure robustness to train-test distribution gaps, [34] corrupted CIFAR-100 test images into CIFAR100-c (more details in Appendix 6.4). As in Puzzle-Mix [41], we report WRN-28-10 results with and without AugMix [35], a pixels data augmentation technique specifically introduced for this task. Tab. 4 shows that Cut-MixMo $(b=4)$ best complements AugMix and reaches $71.1 \%$ Top1.

Table 4: Robustness comparison on CIFAR-100-c.

\begin{tabular}{|c|c|c|c|c|c|c|c|c|c|c|c|c|}
\hline \multirow{2}{*}{$\begin{array}{c}\text { Approach } \\
\text { AugMix }\end{array}$} & \multicolumn{2}{|c|}{1 Net. } & \multirow{2}{*}{$\begin{array}{c}\text { CutMix } \\
-\end{array}$} & \multicolumn{2}{|c|}{ Puzzle-Mix $^{\dagger}$} & \multicolumn{2}{|c|}{ DE (2 Nets) } & \multirow{2}{*}{$\begin{array}{c}\text { MIMO } \\
-\end{array}$} & \multicolumn{2}{|c|}{ Linear-MixMo } & \multicolumn{2}{|c|}{ Cut-MixMo } \\
\hline & - & $\checkmark$ & & - & $\checkmark$ & - & $\checkmark$ & & 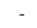 & $\checkmark$ & & \\
\hline To & 52.2 & 7.8 & 51.93 & 58.09 & 70.46 & 53.8 & 69.9 & 53.6 & 556 & 70.4 & 7.0 & 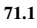 \\
\hline & 73 & 87 & & & & & & & & & 4 & 5 \\
\hline NLL $\downarrow$ & 2.50 & 1.38 & 2.13 & 1.96 & 1.34 & 2.27 & 1.24 & 2.66 & 2.33 & 1.22 & 2.04 & 1.16 \\
\hline
\end{tabular}




\subsection{Pushing MixMo further: Tiny ImageNet}

At a larger scale and with more varied $64 \times 64$ images, Cut-MixMo reaches a new state of the art of $70.24 \%$ on Tiny ImageNet [10] in Tab. 5. We re-use the hyperparameters given in previous state of the art Puzzle-Mix [41]. With $w=1$, PreActResNet-18 [32] is not sufficiently parametrized for MixMo's advantages to express themselves on this challenging dataset. MixMo's full potential shines with wider networks: with $w=2$ and $44.9 \mathrm{M}$ parameters, Cut-MixMo reaches $(69.13 \%, 1.28)$ vs. $(67.76 \%, 1.33)$ for CutMix. Compared to DE with 3 networks, Cut-MixMo performs \{worse, similarly, better $\}$ for width $w \in\{1,2,3\}$. At (almost) the same numbers of parameters, Cut-MixMo when $w=2$ performs better $(69.13 \%, 1.28)$ than DE with 4 networks when $w=1(67.51 \%, 1.31)$.

Table 5: Results: PreActResNet-18- $w$ on Tiny ImageNet.

\begin{tabular}{|c|c|c|c|c|c|c|c|}
\hline \multicolumn{2}{|c|}{ Width $w$ (\# params) } & \multicolumn{2}{|c|}{$w=1(11.2 \mathrm{M})$} & \multicolumn{2}{|c|}{$w=2(44.9 \mathrm{M})$} & \multicolumn{2}{|c|}{$w=3(100.5 \mathrm{M})$} \\
\hline Approach & $\begin{array}{l}\text { Time } \\
\text { Tr./Inf. }\end{array}$ & $\begin{array}{l}\text { Top1 } \\
\%, \uparrow\end{array}$ & $\begin{array}{c}\mathrm{NLL}_{c} \\
\downarrow\end{array}$ & $\begin{array}{l}\text { Top1 } \\
\%, \uparrow\end{array}$ & $\begin{array}{c}\mathrm{NLL}_{c} \\
\downarrow\end{array}$ & $\begin{array}{l}\text { Top1 } \\
\%, \uparrow\end{array}$ & $\begin{array}{c}\mathrm{NLL}_{c} \\
\downarrow\end{array}$ \\
\hline Vanilla & & 62.56 & 1.53 & 64.80 & 1.51 & 65.78 & 1.53 \\
\hline Mixup & & 63.74 & 1.62 & 66.62 & 1.50 & 67.27 & 1.51 \\
\hline Manifold Mixup $^{\dagger}$ & $1 / 1$ & 58.70 & 1.92 & - & - & - & - \\
\hline Co-Mixup $^{\dagger}$ & & 64.15 & - & - & - & - & - \\
\hline CutMix & & 65.09 & 1.58 & 67.76 & 1.33 & 68.95 & 1.29 \\
\hline Puzzle-Mix ${ }^{\dagger}$ & $2 / 1$ & 64.48 & 1.65 & - & - & - & - \\
\hline DE (2 Nets) & $2 / 2$ & 65.53 & 1.39 & 68.06 & 1.37 & 68.38 & 1.36 \\
\hline DE (3 Nets) & $3 / 3$ & 66.76 & 1.34 & 69.05 & 1.29 & 69.36 & 1.28 \\
\hline DE (4 Nets) & $4 / 4$ & 67.51 & 1.31 & 69.94 & 1.24 & 69.72 & 1.26 \\
\hline Linear-MixMo & $2 / 1$ & 61.58 & 1.61 & 66.62 & 1.41 & 68.18 & 1.36 \\
\hline Cut-MixMo & 271 & 63.78 & 1.48 & 68.30 & 1.30 & 69.89 & 1.26 \\
\hline Linear-MixMo & $4 / 1$ & 62.91 & 1.51 & 67.03 & 1.41 & 68.38 & 1.38 \\
\hline Cut-MixMo & $4 / 1$ & 64.44 & 1.48 & 69.13 & 1.28 & 70.24 & 1.19 \\
\hline
\end{tabular}

\subsection{Ensemble of MixMo}

Since MixMo adds very little parameters $(\approx+1 \%)$, we can combine independently trained MixMo like in DE. This ensembling of ensemble of subnetworks leads in practice to the averaging of $M \times N=2 \times N$ predictions. Fig. $10 \mathrm{com}$ pares ensembling for vanilla networks and Cut-MixMo on CIFAR-100. We first recover the Memory Split Advantage [9, 50, 77, 88] (MSA): at similar parameter counts, $N=5$ vanilla WRN-28-3 do better than a single vanilla WRN-28$7\left(+0.10\right.$ in $\left.\mathrm{NLL}_{c}\right)$. Cut-MixMo challenges this MSA: we bridge the gap between using one network or several smaller networks ( -0.04 on same setup). Visually, Cut-MixMo's curves remain closer to the lower envelope: performances are less dependent on how the memory budget is split. This is because Cut-MixMo is effective mainly for larger architectures by better leveraging their parameters.

We also recover that wide vanilla networks tend to be less diverse [55], and thus gain less from ensembling [50]: $N=2$ vanilla WRN-28-14 (83.47\% Top1, $\left.0.656 \mathrm{NLL}_{c}\right)$

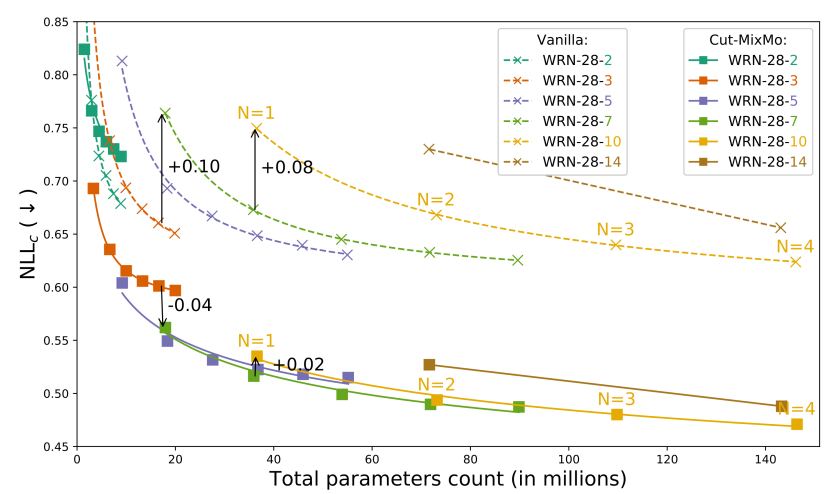

Figure 10: Ensemble effectiveness ( $\mathrm{NLL}_{c}$ /\#params), for different widths $w$ in WRN-28- $w$ and numbers of members $N$. Standard data augmentations on CIFAR-100 with $b=4$. Curves interpolated through power laws [50].

perform not much better than $N=2$ WRN-28-7 (82.94\%, 0.673). Contrarily, Cut-MixMo facilitates the ensembling of large networks with $(86.58 \%, 0.488)$ vs. $(85.50 \%$, 0.516) (more comparisons in Appendix 6.10.

When combined with CutMix [83], Cut-MixMo previously set a new state of the art of $85.77 \%$ with $N=1$ WRN28-10. Final Tab. 6 shows it further reaches $86.63 \%$ with $N=2$ and even $86.81 \%$ with $N=3$.

Table 6: Best results for WRN-28-10 on CIFAR-100 via Cut-MixMo + CutMix [83] $+N$-ensembling and $b=4$. Recent Top1 SoTAs: 85.23 [63], 85.51 [82], 85.74 [88].

\begin{tabular}{cc|ccc|ccc}
\hline$N$ & \# params & Top1 $\uparrow$ & $\begin{array}{c}\text { Average } \\
\text { Top5 } \uparrow\end{array}$ & $\mathrm{NLL}_{c} \downarrow$ & Top1 $\uparrow$ & $\begin{array}{c}\text { Best run } \\
\text { Top5 } \uparrow\end{array}$ & NLL $_{c} \downarrow$ \\
\hline 1 & $36.6 \mathrm{M}$ & $85.77 \pm 0.14$ & $97.36 \pm 0.02$ & $0.524 \pm 0.005$ & 85.92 & 97.36 & 0.518 \\
2 & $73.2 \mathrm{M}$ & $86.63 \pm 0.19$ & $97.73 \pm 0.05$ & $0.479 \pm 0.003$ & 86.75 & 97.80 & 0.475 \\
3 & $109.8 \mathrm{M}$ & $86.81 \pm 0.17$ & $97.85 \pm 0.04$ & $0.464 \pm 0.002$ & 86.94 & 97.83 & 0.464 \\
\hline
\end{tabular}

\section{Conclusion}

We introduce the MixMo framework that generalizes the multi-input multi-output ensembling strategy. MixMo can be analyzed as either an ensembling method or a mixed samples data augmentation, while remaining complementary to works from both lines of research. Finally, MixMo better exploits wide networks and improves the state of the art on CIFAR-100, CIFAR-100-c and Tiny ImageNet.

\section{Acknowledgments}

This work was performed using HPC resources from GENCI-IDRIS (Grant 2021-AD011012262), with financial supports from the ANR agency in the chair VISA-DEEP (ANR-20-CHIA-0022-01), and from Rémy's CIFRE grant between Thales Land and Air Systems and Sorbonne University. We thank Andrei Bursuc for his detailed feedbacks. 


\section{References}

[1] Matti Aksela. Comparison of classifier selection methods for improving committee performance. In MCS, 2003.

[2] Arsenii Ashukha, Alexander Lyzhov, Dmitry Molchanov, and Dmitry Vetrov. Pitfalls of in-domain uncertainty estimation and ensembling in deep learning. In ICLR, 2020.

[3] Kyungjune Baek, Duhyeon Bang, and Hyunjung Shim. Gridmix: Strong regularization through local context mapping. Pattern Recognition, 2021.

[4] Leo Breiman. Bagging predictors. Machine learning, 1996.

[5] Luigi Carratino, Moustapha Cissé, Rodolphe Jenatton, and Jean-Philippe Vert. On mixup regularization. ArXiv preprint, 2020.

[6] Rich Caruana. Multitask learning. Machine learning, 1997.

[7] John Chen, Samarth Sinha, and Anastasios Kyrillidis. Imclr: Implicit contrastive learning for image classification. ArXiv preprint, 2020.

[8] Zhao Chen, Vijay Badrinarayanan, Chen-Yu Lee, and Andrew Rabinovich. Gradnorm: Gradient normalization for adaptive loss balancing in deep multitask networks. In ICML, 2018.

[9] Nadezhda Chirkova, Ekaterina Lobacheva, and Dmitry P. Vetrov. Deep ensembles on a fixed memory budget: One wide network or several thinner ones? ArXiv preprint, 2020.

[10] Patryk Chrabaszcz, Ilya Loshchilov, and Frank Hutter. A downsampled variant of imagenet as an alternative to the cifar datasets. ArXiv preprint, 2017.

[11] Ekin Dogus Cubuk, Barret Zoph, Jon Shlens, and Quoc Le. Randaugment: Practical automated data augmentation with a reduced search space. In NeurIPS, 2020.

[12] J. Deng, W. Dong, R. Socher, L.-J. Li, K. Li, and L. FeiFei. Imagenet: A large-scale hierarchical image database. In CVPR, 2009.

[13] Terrance DeVries and Graham W Taylor. Improved regularization of convolutional neural networks with cutout. ArXiv preprint, 2017.

[14] Thomas G Dietterich. Ensemble methods in machine learning. In MCS, 2000.

[15] Nikita Durasov, Timur Bagautdinov, Pierre Baque, and Pascal Fua. Masksembles for uncertainty estimation. ArXiv preprint, 2020.

[16] Michael Dusenberry, Ghassen Jerfel, Yeming Wen, Yian Ma, Jasper Snoek, Katherine Heller, Balaji Lakshminarayanan, and Dustin Tran. Efficient and scalable bayesian neural nets with rank-1 factors. In ICML, 2020.

[17] Mojtaba Faramarzi, Mohammad Amini, Akilesh Badrinaaraayanan, Vikas Verma, and Sarath Chandar. Patchup: A regularization technique for convolutional neural networks. ArXiv preprint, 2020.

[18] Stanislav Fort, Huiyi Hu, and Balaji Lakshminarayanan. Deep ensembles: A loss landscape perspective. ArXiv preprint, 2019.

[19] Jonathan Frankle and Michael Carbin. The lottery ticket hypothesis: Finding sparse, trainable neural networks. In ICLR, 2019.
[20] Geoff French, Timo Aila, Samuli Laine, Michal Mackiewicz, and Graham Finlayson. Semi-supervised semantic segmentation needs strong, high-dimensional perturbations. ArXiv preprint, 2019.

[21] Geoff French, Avital Oliver, and Tim Salimans. Milking cowmask for semi-supervised image classification. ArXiv preprint, 2020.

[22] Yarin Gal and Zoubin Ghahramani. Dropout as a bayesian approximation: Representing model uncertainty in deep learning. In $I C M L, 2016$.

[23] Yuan Gao, Zixiang Cai, and Lei Yu. Intra-ensemble in neural networks. ArXiv preprint, 2019.

[24] Golnaz Ghiasi, Tsung-Yi Lin, and Quoc V Le. Dropblock: A regularization method for convolutional networks. In NeurIPS, 2018.

[25] Chuan Guo, Geoff Pleiss, Yu Sun, and Kilian Q. Weinberger. On calibration of modern neural networks. In ICML, 2017.

[26] Hongyu Guo, Yongyi Mao, and Richong Zhang. Mixup as locally linear out-of-manifold regularization. In AAAI, 2019.

[27] Fredrik K Gustafsson, Martin Danelljan, and Thomas B Schon. Evaluating scalable bayesian deep learning methods for robust computer vision. In CVPR Workshops, 2020.

[28] Lars Kai Hansen and Peter Salamon. Neural network ensembles. IEEE transactions on pattern analysis and machine intelligence, 1990.

[29] Ethan Harris, Antonia Marcu, Matthew Painter, Mahesan Niranjan, Adam Prügel-Bennett, and Jonathon Hare. Fmix: Enhancing mixed sample data augmentation. ArXiv preprint, 2020.

[30] Marton Havasi, Rodolphe Jenatton, Stanislav Fort, Jeremiah Liu, Jasper Roland Snoek, Balaji Lakshminarayanan, Andrew Mingbo Dai, and Dustin Tran. Training independent subnetworks for robust prediction. In ICLR, 2021.

[31] Kaiming He, Xiangyu Zhang, Shaoqing Ren, and Jian Sun. Deep residual learning for image recognition. In $C V P R$, 2016.

[32] Kaiming He, Xiangyu Zhang, Shaoqing Ren, and Jian Sun. Identity mappings in deep residual networks. In $E C C V$, 2016.

[33] Zhuoxun He, Lingxi Xie, Xin Chen, Ya Zhang, Yanfeng Wang, and Qi Tian. Data augmentation revisited: Rethinking the distribution gap between clean and augmented data. ArXiv preprint, 2019.

[34] Dan Hendrycks and Thomas Dietterich. Benchmarking neural network robustness to common corruptions and perturbations. In ICLR, 2019.

[35] Dan Hendrycks, Norman Mu, Ekin Dogus Cubuk, Barret Zoph, Justin Gilmer, and Balaji Lakshminarayanan. Augmix: A simple data processing method to improve robustness and uncertainty. In ICLR, 2019.

[36] Elad Hoffer, Tal Ben-Nun, Itay Hubara, Niv Giladi, Torsten Hoefler, and Daniel Soudry. Augment your batch: Improving generalization through instance repetition. In CVPR, 2020.

[37] Gao Huang, Yixuan Li, Geoff Pleiss, Zhuang Liu, John E. Hopcroft, and Kilian Q Weinberger. Snapshot ensembles: Train 1, get $\mathrm{m}$ for free. In ICLR, 2017. 
[38] Hiroshi Inoue. Data augmentation by pairing samples for images classification. ArXiv preprint, 2018.

[39] Pavel Izmailov, Dmitrii Podoprikhin, Timur Garipov, Dmitry Vetrov, and Andrew Gordon Wilson. Averaging weights leads to wider optima and better generalization. In $U A I$, 2018.

[40] JangHyun Kim, Wonho Choo, Hosan Jeong, and Hyun Oh Song. Co-mixup: Saliency guided joint mixup with supermodular diversity. In ICLR, 2021.

[41] Jang-Hyun Kim, Wonho Choo, and Hyun Oh Song. Puzzle mix: Exploiting saliency and local statistics for optimal mixup. In ICML, 2020.

[42] Alex Krizhevsky and Geoffrey Hinton. Learning multiple layers of features from tiny images. Technical report, 2009.

[43] Balaji Lakshminarayanan, Alexander Pritzel, and Charles Blundell. Simple and scalable predictive uncertainty estimation using deep ensembles. In NeurIPS, 2017.

[44] Yann Lecun, J. S. Denker, Sara A. Solla, R. E. Howard, and L.D. Jackel. Optimal brain damage. In NeurIPS, 1990.

[45] Stefan Lee, Senthil Purushwalkam, Michael Cogswell, David J. Crandall, and Dhruv Batra. Why M heads are better than one: Training a diverse ensemble of deep networks. ArXiv preprint, 2015.

[46] Boyi Li, Felix Wu, Ser-Nam Lim, Serge Belongie, and Kilian Q. Weinberger. On feature normalization and data augmentation. ArXiv preprint, 2020.

[47] Hao Li, Asim Kadav, Igor Durdanovic, Hanan Samet, and Hans Peter Graf. Pruning filters for efficient convnets. In ICLR, 2017.

[48] Daojun Liang, Feng Yang, Tian Zhang, and Peter Yang. Understanding mixup training methods. IEEE Access, 2018.

[49] Tsung-Yi Lin, Priya Goyal, Ross Girshick, Kaiming He, and Piotr Dollár. Focal loss for dense object detection. In ICCV, 2017.

[50] Ekaterina Lobacheva, Nadezhda Chirkova, Maxim Kodryan, and Dmitry P Vetrov. On power laws in deep ensembles. In NeurIPS, 2020.

[51] Raphael Gontijo Lopes, Sylvia J. Smullin, Ekin D. Cubuk, and Ethan Dyer. Affinity and diversity: Quantifying mechanisms of data augmentation. In CoRR, 2020.

[52] Pavlo Molchanov, Stephen Tyree, Tero Karras, Timo Aila, and Jan Kautz. Pruning convolutional neural networks for resource efficient transfer learning. In ICLR, 2017.

[53] Rafael Müller, Simon Kornblith, and Geoffrey E Hinton. When does label smoothing help? In NeurIPS, 2019.

[54] Mahdi Pakdaman Naeini, Gregory Cooper, and Milos Hauskrecht. Obtaining well calibrated probabilities using bayesian binning. In $A A A I, 2015$.

[55] Brady Neal, Sarthak Mittal, Aristide Baratin, Vinayak Tantia, Matthew Scicluna, Simon Lacoste-Julien, and Ioannis Mitliagkas. A modern take on the bias-variance tradeoff in neural networks. ArXiv preprint, 2018.

[56] Jeremy Nixon, Michael W Dusenberry, Linchuan Zhang, Ghassen Jerfel, and Dustin Tran. Measuring calibration in deep learning. In CVPR workshop, 2019.

[57] David Opitz and Richard Maclin. Popular ensemble methods: An empirical study. Journal of artificial intelligence research, 1999.
[58] Yaniv Ovadia, Emily Fertig, Jie Ren, Zachary Nado, David Sculley, Sebastian Nowozin, Joshua Dillon, Balaji Lakshminarayanan, and Jasper Snoek. Can you trust your model's uncertainty? evaluating predictive uncertainty under dataset shift. In NeurIPS, 2019.

[59] Adam Paszke, Sam Gross, Francisco Massa, Adam Lerer, James Bradbury, Gregory Chanan, Trevor Killeen, Zeming Lin, Natalia Gimelshein, Luca Antiga, Alban Desmaison, Andreas Kopf, Edward Yang, Zachary DeVito, Martin Raison, Alykhan Tejani, Sasank Chilamkurthy, Benoit Steiner, Lu Fang, Junjie Bai, and Soumith Chintala. Pytorch: An imperative style, high-performance deep learning library. In NeurIPS. 2019.

[60] Ankit Pensia, Shashank Rajput, Alliot Nagle, Harit Vishwakarma, and Dimitris Papailiopoulos. Optimal lottery tickets via subsetsum: Logarithmic over-parameterization is sufficient. In NeurIPS, 2020.

[61] Gabriel Pereyra, George Tucker, Jan Chorowski, Łukasz Kaiser, and Geoffrey Hinton. Regularizing neural networks by penalizing confident output distributions. In ICLR workshop, 2017.

[62] Michael Perrone and Leon Cooper. When networks disagree: Ensemble methods for hybrid neural networks. Neural networks for speech and image processing, 1993.

[63] Jie Qin, Jiemin Fang, Qian Zhang, Wenyu Liu, Xingang Wang, and Xinggang Wang. Resizemix: Mixing data with preserved object information and true labels. ArXiv preprint, 2020.

[64] Alexandre Rame and Matthieu Cord. Dice: Diversity in deep ensembles via conditional redundancy adversarial estimation. In ICLR, 2021.

[65] Ramprasaath R Selvaraju, Michael Cogswell, Abhishek Das, Ramakrishna Vedantam, Devi Parikh, and Dhruv Batra. Grad-cam: Visual explanations from deep networks via gradient-based localization. In ICCV, 2017.

[66] Masoumeh Soflaei, Hongyu Guo, Ali Al-Bashabsheh, Yongyi Mao, and Richong Zhang. Aggregated learning: A vector-quantization approach to learning neural network classifiers. In AAAI, 2020.

[67] Nitish Srivastava, Geoffrey Hinton, Alex Krizhevsky, Ilya Sutskever, and Ruslan Salakhutdinov. Dropout: a simple way to prevent neural networks from overfitting. Journal of Machine Learning Research, 2014.

[68] Cecilia Summers and Michael J Dinneen. Improved mixedexample data augmentation. In WACV, 2019.

[69] Christian Szegedy, Wei Liu, Yangqing Jia, Pierre Sermanet, Scott Reed, Dragomir Anguelov, Dumitru Erhan, Vincent Vanhoucke, and Andrew Rabinovich. Going deeper with convolutions. In $C V P R, 2015$.

[70] Ryo Takahashi, Takashi Matsubara, and Kuniaki Uehara. Data augmentation using random image cropping and patching for deep cnns. IEEE Transactions on Circuits and Systems for Video Technology, 2020.

[71] Naftali Tishby. The information bottleneck method. In Allerton Conference on Communication, Control and Computation, 2001. 
[72] Yuji Tokozume, Yoshitaka Ushiku, and Tatsuya Harada. Between-class learning for image classification. In $C V P R$, 2018.

[73] Yuji Tokozume, Yoshitaka Ushiku, and Tatsuya Harada. Learning from between-class examples for deep sound recognition. In ICLR, 2018.

[74] A. F. M. Shahab Uddin, Mst. Sirazam Monira, Wheemyung Shin, TaeChoong Chung, and Sung-Ho Bae. Saliencymix: A saliency guided data augmentation strategy for better regularization. In ICLR, 2021.

[75] Andreas Veit, Michael Wilber, and Serge Belongie. Residual networks behave like ensembles of relatively shallow networks. In NeurIPS, 2016.

[76] Vikas Verma, Alex Lamb, Christopher Beckham, Amir Najafi, Ioannis Mitliagkas, David Lopez-Paz, and Yoshua Bengio. Manifold mixup: Better representations by interpolating hidden states. In ICML, 2019.

[77] Xiaofang Wang, Dan Kondratyuk, Kris M. Kitani, Yair Movshovitz-Attias, and Elad Eban. Multiple networks are more efficient than one: Fast and accurate models via ensembles and cascades. ArXiv preprint, 2020.

[78] Abdul Wasay, Brian Hentschel, Yuze Liao, Sanyuan Chen, and Stratos Idreos. Mothernets: Rapid deep ensemble learning. In MLSys, 2020.

[79] Yeming Wen, Ghassen Jerfel, Rafael Muller, Michael W Dusenberry, Jasper Snoek, Balaji Lakshminarayanan, and Dustin Tran. Combining ensembles and data augmentation can harm your calibration. In ICLR, 2021.

[80] Andrew Gordon Wilson and Pavel Izmailov. Bayesian deep learning and a probabilistic perspective of generalization. NeurIPS, 2020.

[81] Yoichi Yaguchi, Fumiyuki Shiratani, and Hidekazu Iwaki. Mixfeat: Mix feature in latent space learns discriminative space. Openreview preprint, 2019.

[82] Taojiannan Yang, Sijie Zhu, and Chen Chen. Gradaug: A new regularization method for deep neural networks. NeurIPS, 2020.

[83] Sangdoo Yun, Dongyoon Han, Seong Joon Oh, Sanghyuk Chun, Junsuk Choe, and Youngjoon Yoo. Cutmix: Regularization strategy to train strong classifiers with localizable features. In ICCV, 2019.

[84] Sergey Zagoruyko and Nikos Komodakis. Wide residual networks. In BMVC, 2016.

[85] Chiyuan Zhang, Samy Bengio, Moritz Hardt, Benjamin Recht, and Oriol Vinyals. Understanding deep learning requires rethinking generalization. ICLR, 2017.

[86] Hongyi Zhang, Moustapha Cisse, Yann N Dauphin, and David Lopez-Paz. mixup: Beyond empirical risk minimization. In ICLR, 2018.

[87] Linjun Zhang, Zhun Deng, Kenji Kawaguchi, Amirata Ghorbani, and James Zou. How does mixup help with robustness and generalization? ICLR, 2021.

[88] Shuai Zhao, Liguang Zhou, Wenxiao Wang, Deng Cai, Tin Lun Lam, and Yangsheng Xu. Splitnet: Divide and cotraining. ArXiv preprint, 2020. 


\section{Appendix}

The sections in this Appendix follow a similar order to their related sections in the main paper. We first illustrate the reweighting of the loss components in Appendix 6.1 Appendix 6.2 elaborates on our analysis of filters activity. Appendix 6.3 clarifies our framework generalization with $M>2$ subnetworks. We describe in greater details our implementation in Appendix 6.4, and then our evaluation setting in 6.5. Appendix 6.6 showcases training dynamics. We provide a quick refresher on common MSDA techniques in Appendix 6.7. Appendix 6.8 studies the importance of $\alpha$. Appendix 6.9 is a preliminary study of MixMo on ImageNet. Appendix 6.10 analyzes ensembles of Cut-MixMo with CutMix that reach state of the art. Finally, we provide a pseudocode in Algorithm 1

\subsection{Weighting function $w_{r}$}

As outlined in Section 3.3 the asymmetry in the mixing mechanism leads to asymmetry in the relative importance of the two inputs. Thus we reweight the loss components with function $w_{r}$, defined as $w_{r}(\kappa)=2 \frac{\kappa^{1 / r}}{\kappa^{1 / r}+(1-\kappa)^{1 / r}}$. It rescales the mixing ratio $\kappa$ through the use of a $\frac{1}{r}$ root operator. In the main paper, we have focused on $r=3$.

Fig. 11 illustrates how $w_{r}$ behaves for $r \in\{1,2,3,4,10\}$ and $r \rightarrow \infty$. The first extreme $r=1$ matches the diagonal $w_{r}(\kappa)=2 \kappa$, without rescaling of $\kappa$, similarly to what is customary in MSDA. Our experiments in Section 4.3.3 justified the initial idea to shift the weighting function closer to the horizontal and constant curve $w_{r}(\kappa)=1$ with higher $r$. In the other experiments, we always set $r=3$.

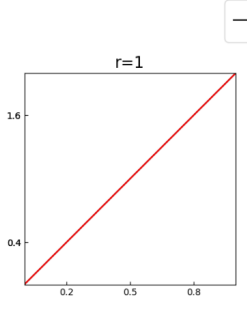

$$
x \mapsto 2 x \quad x \mapsto w_{r}(x)
$$
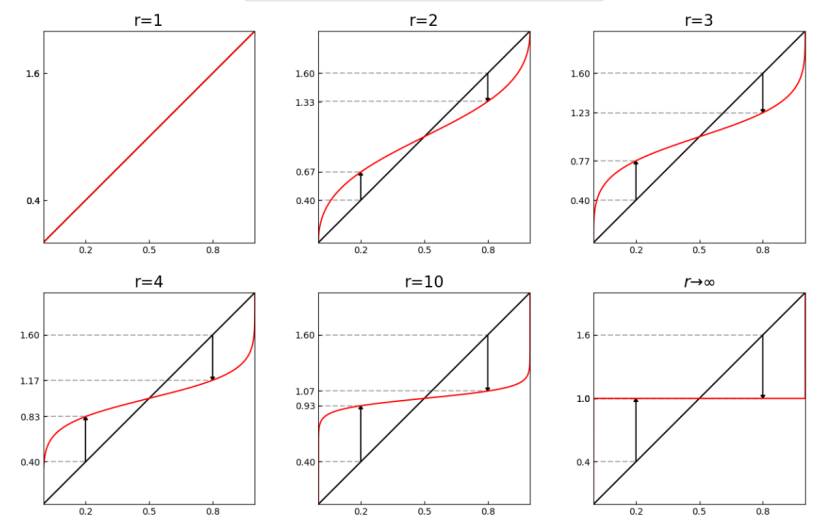

Figure 11: Curves of the reweighting operation that projects $\kappa$ to the flattened ratio $w_{r}(\kappa)$

\subsection{Filters activity}

We argued in Section 3.4 that MixMo better leverages additional parameters in wider networks. Concretely, a
Table 7: Proportion (\%) of active filters in core network vs. width $w$ for a WRN-28- $w$ on CIFAR 100 and different activity thresholds $t_{a}$.

\begin{tabular}{cc|cccc}
\hline Method & Width & $t_{a}=0.2$ & $t_{a}=0.3$ & $t_{a}=0.4$ & $t_{a}=0.5$ \\
\hline \multirow{6}{*}{ Vanilla } & 2 & 98.9 & 98.8 & 97.8 & 93.3 \\
& 3 & 97.3 & 96.4 & 93.2 & 87.5 \\
& 4 & 96.5 & 95.2 & 91.2 & 81.6 \\
& 5 & 95.1 & 91.7 & 85.7 & 73.3 \\
& 7 & 92.6 & 88.2 & 81.0 & 69.5 \\
& 10 & 87.8 & 80.4 & 71.5 & 57.3 \\
& 14 & 83.9 & 74.0 & 61.6 & 46.8 \\
\hline \multirow{6}{*}{ CutMix } & 2 & 99.2 & 99.0 & 97.8 & 95.3 \\
& 3 & 98.7 & 98.5 & 97.2 & 93.4 \\
& 4 & 98.1 & 97.4 & 94.0 & 87.3 \\
& 5 & 97.0 & 96.1 & 90.7 & 80.6 \\
& 7 & 95.8 & 94.0 & 86.2 & 74.6 \\
& 10 & 93.5 & 88.4 & 81.3 & 67.0 \\
Cut-MixMo & 14 & 89.4 & 81.9 & 70.3 & 50.9 \\
\hline \multirow{6}{*}{} & 2 & 100.0 & 100.0 & 99.4 & 97.3 \\
& 3 & 99.8 & 99.8 & 99.7 & 98.7 \\
& 4 & 99.7 & 99.7 & 99.6 & 98.7 \\
& 5 & 99.3 & 99.3 & 98.9 & 97.4 \\
& 10 & 98.9 & 98.8 & 98.0 & 95.2 \\
& 14 & 98.5 & 98.2 & 96.8 & 92.4 \\
& & & & &
\end{tabular}

larger proportion of filters in large networks really help for classification as demonstrated in Fig. $4 \mathrm{a}$ and $4 \mathrm{~b}$ in the main paper. Following common practices in the structured pruning literature [47], we used the $l_{1}$-norm of convolutional filters as a proxy for importance. These 3D filters are of shape $n_{i} \times k \times k$ with $n_{i}$ the number of input channels and $k$ the kernel size. In Fig. $4 \mathrm{~b}$, we arbitrarily defined a filter as active if its $l_{1}$-norm is at least $40 \%$ of the highest filter $l_{1}$-norm in that filter's layer. We report the average percentage of active filters across all filters in the core network $\mathcal{C}$, for 3 learning strategies: vanilla, CutMix and Cut-MixMo.

The threshold $t_{a}=0.4$ was chosen for visualization purposes. Nevertheless, the observed trend in activity proportions remains for varying thresholds in Tab.7. For example, for the lax $t_{a}=0.2$, CutMix uses $93.5 \%$ of filters vs. $98.5 \%$ for Cut-MixMo.

\subsection{Generalization to $M>2$ heads}

We have mostly discussed our MixMo framework with $M=2$ subnetworks. For better readability, we referred to the mixing ratios $\kappa$ and $1-\kappa$ with $\kappa \sim$ $\operatorname{Beta}(\alpha, \alpha)$. It's equivalent to a more generic formulation $\left(\kappa_{0}, \kappa_{1}\right) \in \operatorname{Dir}_{2}(\alpha)$ from a symmetric Dirichlet distribution with concentration parameter $\alpha$. This leads to the alternate equations $\mathcal{L}_{\text {MixMo }}=\sum_{i=0,1} w_{r}\left(\kappa_{i}\right) \mathcal{L}_{\mathrm{CE}}\left(y_{i}, \hat{y}_{i}\right)$, where $w_{r}\left(\kappa_{i}\right)=2 \frac{\kappa_{i}^{1 / r}}{\sum_{j=0,1} \kappa_{j}^{1 / r}}$.

Now generalization to the general case $M \geq 2$ is straightforward. We draw a tuple $\left\{\kappa_{i}\right\}_{0 \leq i<M} \sim \operatorname{Dir}_{M}(\alpha)$ 
and optimize the training loss:

$$
\mathcal{L}_{\text {MixMo }}=\sum_{i=0}^{M-1} w_{r}\left(\kappa_{i}\right) \mathcal{L}_{\mathrm{CE}}\left(y_{i}, \hat{y}_{i}\right),
$$

where the new weighting naturally follows:

$$
w_{r}\left(\kappa_{i}\right)=M \frac{\kappa_{i}^{1 / r}}{\sum_{j=0}^{M-1} \kappa_{j}^{1 / r}}, \forall i \in\{0, \ldots, M-1\} .
$$

The remaining point is the generalization of the mixing block $\mathcal{M}$, that relies on the existence of MSDA methods for $M>2$ inputs. The linear interpolation can be easily expanded as in Mixup:

$$
\mathcal{M}_{\text {Linear-MixMo }}\left(\left\{l_{i}\right\}\right)=M \sum_{i=0}^{M-1} \kappa_{i} l_{i},
$$

where $l_{i}=c_{i}\left(x_{i}\right)$. However, extensions for other masking MSDAs have only recently started to emerge [40]. For example, CutMix is not trivially generalizable to $M>2$, as the patches could overlap and hide important semantic components. In our experiments, a soft extension of CutMixMo performs best: it first linearly interpolates $M-1$ inputs and then patches a region from the $M$-th:

$$
\begin{aligned}
\mathcal{M}_{\text {Cut-MixMo }}\left(\left\{l_{i}\right\}\right) & =M\left[\mathbb{1}_{\mathcal{M}} \odot l_{k}+\right. \\
& \left.\left(\mathbb{1}-\mathbb{1}_{\mathcal{M}}\right) \odot \sum_{i=0, i \neq k}^{M-1} \frac{\kappa_{i}}{1-\kappa_{k}} l_{i}\right],
\end{aligned}
$$

where $\mathbb{1}_{\mathcal{M}}$ is a rectangle of area ratio $\kappa_{k}$ and $k$ sampled uniformly in $\{0,1, \ldots, M-1\}$. However, it has been less successful than $M=2$, as only two subnetworks can fit independently in standard parameterization regimes. Future work could design new framework components, such as specific mixing blocks, to tackle these limits.

\subsection{Implementation details}

We first used the popular image classification datasets CIFAR-100 and CIFAR-10 [42]. They contain 60k $32 \times 32$ natural and colored images in respectively 100 classes and 10 classes, with 50k training images and 10k test images. At a larger scale, we study Tiny ImageNet [10], a downsampled version of ImageNet [12]. It contains 200 different categories, $100 \mathrm{k} 64 \times 64$ training images (i.e. 500 images per class) and 10k test images.

Our code was adapted from the official MIMO [30] implementation 1 For CIFAR, we re-use the hyperparameters from MIMO [30]. The optimizer is SGD with learning rate of $\frac{0.1}{b} \times \frac{\text { batch-size }}{128}$, batch size 64 , linear warmup over 1 epoch, decay rate 0.1 at steps $\{100,200,225\}, l_{2}$

\footnotetext{
1https://github.com/google/edward2/
}

regularization 3e-4. We follow standard MSDA practices $[2,41,83]$ and set the maximum number of epochs to 300 . For Tiny ImageNet, we adapt PreActResNet-18-w, with $w \in\{1,2,3\}$ times more filters. We re-use the hyperparameters from Puzzle-Mix [41]. The optimizer is SGD with learning rate of $\frac{0.2}{b}$, batch size 100 , decay rate 0.1 at steps $\{600,900\}, 1200$ epochs maximum, weight decay 1e4. Our experiments ran on a single NVIDIA 12Go-TITAN $X$ Pascal GPU. All results without a $\dagger$ were obtained with these training configurations. We will soon release our code and pre-trained models to facilitate reproducibility.

Batch repetition increases performances at the cost of longer training, which may be discouraging for some practitioners. Thus in addition to $b=4$ as in MIMO [30], we often consider the quicker $b=2$. Note that most of our concurrent approaches also increase training time: DE [43] via several independent trainings, Puzzle-Mix [41] via saliency detection $(\approx \times 2)$, GradAug [82] via multiple subnetworks predictions $(\approx \times 3)$ or Mixup BA [36] via 10 batch augmentations $(\approx \times 7$ with our hardware on a single GPU).

MixMo operates in the features space and is complementary with pixels augmentations, i.e. cropping, AugMix. The standard vanilla pixels data augmentation [31] consists of 4 pixels padding, random cropping and horizontal flipping. When combined with CutMix, notably to benefit from multilabel smoothing, the input may be of the form: $\left(m_{x}\left(x_{i}, x_{k}, \lambda\right), x_{j}\right)$, where $x_{k}$ is randomly chosen in the whole dataset, and not only inside the current batch ${ }^{2}$ Moreover, $\mathcal{M}_{\text {Cut-MixMo }}$ modifies by $\mathbb{1}_{\mathcal{M}}$ the visible part from mask $\mathbb{1}_{m}$ (of area $\lambda$ ). We thus modify targets accordingly: $\left(\lambda^{\prime} y_{i}+\left(1-\lambda^{\prime}\right) y_{k}, y_{j}\right)$ where $\lambda^{\prime}=\frac{\sum \mathbb{1}_{m} \odot \mathbb{1}_{\mathcal{M}}}{\sum \mathbb{1}_{\mathcal{M}}}$. To fully benefit from $b$, we force the repeated $x_{i}$ to remain predominant in its $b$ appearances: i.e., we swap $x_{i}$ and $x_{k}$ if $\lambda^{\prime}<0.5$. We see CutMix as a perturbation on the main batch sample.

Distributional uncertainty measures help when there is a mismatch between train and test data distributions. Thus [34] introduced CIFAR-100-c on which AugMix performs best. AugMix sums the pixels from a chain of several augmentations and is complementary to our approach in features. We use default parameter ${ }^{3}$, the severity is set 3, the mixture's width to 3 and the mixture's depth to 4 . We exclude operations in AugMix which overlap with CIFAR100-c corruptions: thus, [equalize, posterize, rotate, solarize, shear_x, shear_y, translate_x, translate_y] remain. We disabled the Jensen-Shannon Divergence loss between predictions for the clean image and for the same image AugMix augmented: that would otherwise triple the training time. For comparison of out-of-domain uncertainty estimations, we report NLL as in [30, 58]: indeed, the recommendation of [2] to apply TS only stands for in-domain test set.

${ }^{2}$ Following https://github.com/ildoonet/cutmix

3 https://github.com/google-research/augmix/ blob/master/cifar.py 

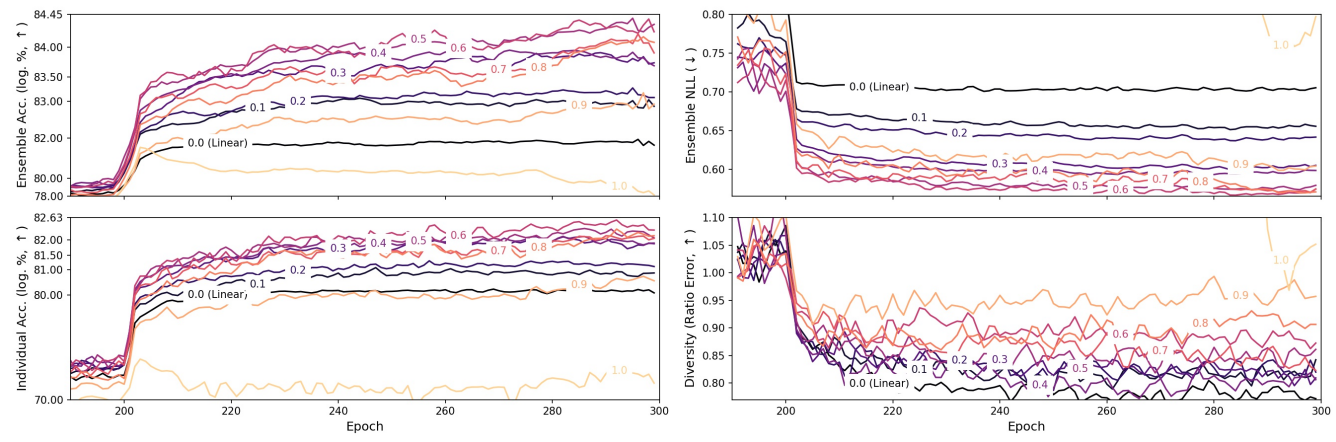

Figure 12: Training dynamics. Higher probability $p$ of binary mixing via patches increases diversity (lower right), and also subnetworks accuracy (lower left) but only up to $p=0.6$. Around this value, we obtain best ensemble performances, in terms of accuracy (upper left) or uncertainty estimation (upper right). $b=2, r=3, \alpha=3$ with WRN-28-10 on CIFAR-100.

Table 8: WRN-28-10 on CIFAR without early stopping.

\begin{tabular}{|c|c|c|c|c|c|c|c|c|c|c|}
\hline \multicolumn{2}{|l|}{ Dataset } & \multicolumn{5}{|c|}{ CIFAR-100 } & \multicolumn{4}{|c|}{ CIFAR-10 } \\
\hline Approach & $\begin{array}{c}\text { Time } \\
\text { Tr./Inf. }\end{array}$ & $\begin{array}{l}\text { Top1 } \\
\%, \uparrow\end{array}$ & $\begin{array}{l}\text { Top5 } \\
\%, \uparrow\end{array}$ & $\begin{array}{l}\mathrm{NLL}_{c} \\
10^{-2}, \downarrow\end{array}$ & $\begin{array}{c}\text { NLL } \\
10^{-2}, \downarrow\end{array}$ & $\begin{array}{c}\text { ECE } \\
10^{-2}, \downarrow\end{array}$ & $\begin{array}{l}\text { Top1 } \\
\%, \uparrow\end{array}$ & $\begin{array}{l}\mathrm{NLL}_{c} \\
10^{-2}, \downarrow\end{array}$ & $\begin{array}{c}\text { NLL } \\
10^{-2}, \downarrow\end{array}$ & $\begin{array}{c}\text { ECE } \\
10^{-2}, \downarrow\end{array}$ \\
\hline $\begin{array}{c}\text { Vanilla } \\
\text { Mixup } \\
\text { Hard PatchUp } \\
\text { CutMix } \\
\end{array}$ & $1 / 1$ & $\begin{array}{l}81.47 \\
83.15 \\
83.87 \\
83.74 \\
\end{array}$ & $\begin{array}{c}95.57 \\
95.75 \\
- \\
96.18 \\
\end{array}$ & $\begin{array}{c}73.6 \\
66.3 \\
- \\
65.4 \\
\end{array}$ & $\begin{array}{l}76.2 \\
67.3 \\
66.0 \\
66.1 \\
\end{array}$ & $\begin{array}{c}6.47 \\
\mathbf{1 . 6 2} \\
- \\
4.95 \\
\end{array}$ & $\begin{array}{l}96.31 \\
97.00 \\
97.47 \\
97.21 \\
\end{array}$ & $\begin{array}{c}12.5 \\
11.3 \\
- \\
9.7 \\
\end{array}$ & $\begin{array}{l}14.1 \\
11.5 \\
11.4 \\
10.8 \\
\end{array}$ & $\begin{array}{c}1.95 \\
0.97 \\
- \\
1.51 \\
\end{array}$ \\
\hline Puzzle-Mix ${ }^{\dagger}$ & $2 / 1$ & 84.05 & 96.08 & 66.9 & 68.1 & 2.76 & - & - & - & - \\
\hline $\begin{array}{l}\text { GradAug }^{\dagger} \\
+ \text { CutMix }^{\dagger}\end{array}$ & $3 / 1$ & $\begin{array}{l}83.98 \\
85.25\end{array}$ & $\begin{array}{l}96.28 \\
96.85\end{array}$ & - & - & $\begin{array}{l}- \\
-\end{array}$ & - & - & - & - \\
\hline Mixup $\mathrm{BA}^{\dagger}$ & $7 / 1$ & 84.30 & - & - & - & - & 97.80 & - & - & - \\
\hline $\begin{array}{l}\text { DE (2 Nets) } \\
+ \text { CutMix }\end{array}$ & $2 / 2$ & $\begin{array}{l}83.15 \\
85.46\end{array}$ & $\begin{array}{l}96.30 \\
96.90\end{array}$ & $\begin{array}{l}66.0 \\
57.4\end{array}$ & $\begin{array}{l}67.2 \\
57.5\end{array}$ & $\begin{array}{l}5.15 \\
3.62\end{array}$ & $\begin{array}{l}96.58 \\
97.51\end{array}$ & $\begin{array}{c}11.1 \\
8.7\end{array}$ & $\begin{array}{l}12.2 \\
9.0\end{array}$ & $\begin{array}{l}1.82 \\
1.16\end{array}$ \\
\hline $\operatorname{MIMO}(M=2)$ & & 82.04 & 95.75 & 69.1 & 72.4 & 6.32 & 96.33 & 12.1 & 13.4 & 1.89 \\
\hline $\begin{array}{l}\text { Linear-MixMo } \\
\quad+\text { CutMix }\end{array}$ & $2 / 1$ & $\begin{array}{l}81.88 \\
84.55\end{array}$ & $\begin{array}{l}95.97 \\
96.95\end{array}$ & $\begin{array}{l}67.8 \\
57.4\end{array}$ & $\begin{array}{l}70.3 \\
57.5\end{array}$ & $\begin{array}{l}6.20 \\
2.54\end{array}$ & $\begin{array}{l}96.55 \\
97.34 \\
\end{array}$ & $\begin{array}{c}11.4 \\
8.9\end{array}$ & $\begin{array}{c}12.5 \\
9.3\end{array}$ & $\begin{array}{l}1.67 \\
1.34\end{array}$ \\
\hline $\begin{array}{l}\text { Cut-MixMo } \\
+ \text { CutMix }\end{array}$ & & $\begin{array}{l}84.07 \\
85.17\end{array}$ & $\begin{array}{l}96.97 \\
97.28\end{array}$ & $\begin{array}{l}56.6 \\
54.4\end{array}$ & $\begin{array}{l}57.9 \\
54.5\end{array}$ & $\begin{array}{l}4.19 \\
2.13\end{array}$ & $\begin{array}{l}97.26 \\
97.33\end{array}$ & $\begin{array}{l}8.7 \\
8.5\end{array}$ & $\begin{array}{l}9.1 \\
8.6\end{array}$ & $\begin{array}{l}0.98 \\
\mathbf{0 . 8 8}\end{array}$ \\
\hline $\begin{array}{c}\operatorname{MIMO}(M=2) \\
\operatorname{MIMO}^{\dagger}(M=3)\end{array}$ & & $\begin{array}{c}82.74 \\
82.0\end{array}$ & $\begin{array}{c}95.90 \\
-\end{array}$ & $\begin{array}{c}67.0 \\
-\end{array}$ & $\begin{array}{l}74.0 \\
69.0\end{array}$ & $\begin{array}{c}7.56 \\
2.2\end{array}$ & $\begin{array}{c}96.66 \\
96.4\end{array}$ & $\begin{array}{c}11.5 \\
-\end{array}$ & $\begin{array}{l}13.6 \\
12.3\end{array}$ & $\begin{array}{c}1.98 \\
1.0\end{array}$ \\
\hline $\begin{array}{l}\text { Linear-MixMo } \\
\quad+\text { CutMix }\end{array}$ & $4 / 1$ & $\begin{array}{l}82.53 \\
85.24\end{array}$ & $\begin{array}{l}96.08 \\
96.97 \\
\end{array}$ & $\begin{array}{r}65.8 \\
56.3 \\
\end{array}$ & $\begin{array}{l}68.5 \\
56.4 \\
\end{array}$ & $\begin{array}{l}6.64 \\
3.53 \\
\end{array}$ & $\begin{array}{l}96.78 \\
97.53 \\
\end{array}$ & $\begin{array}{l}10.8 \\
8.8\end{array}$ & $\begin{array}{l}11.8 \\
8.6\end{array}$ & $\begin{array}{l}1.80 \\
1.19 \\
\end{array}$ \\
\hline $\begin{array}{l}\text { Cut-MixMo } \\
\text { + CutMix }\end{array}$ & & $\begin{array}{l}85.32 \\
\mathbf{8 5 . 5 9}\end{array}$ & $\begin{array}{l}97.12 \\
\mathbf{9 7 . 3 3}\end{array}$ & $\begin{array}{l}53.6 \\
\mathbf{5 3 . 2}\end{array}$ & $\begin{array}{l}54.8 \\
\mathbf{5 3 . 3}\end{array}$ & $\begin{array}{l}4.53 \\
1.95\end{array}$ & $\begin{array}{l}97.42 \\
97.70\end{array}$ & $\begin{array}{l}8.1 \\
8.0\end{array}$ & $\begin{array}{l}8.4 \\
8.2\end{array}$ & $\begin{array}{l}1.15 \\
0.98\end{array}$ \\
\hline
\end{tabular}

\subsection{Evaluation setting and metrics}

We reproduce the experimental setting from CutMix [83], Manifold Mixup [76] and other works such as the recent state-of-the-art ResizeMix [63]: in absence of a validation dataset, results are reported at the epoch that yields the best test accuracy. For fair comparison, we apply this early stopping for all concurrent approaches. Nonetheless, for the sake of completeness, Table 8 shows results without early stopping on the main experiment (CIFAR with a standard WRN-28-10). We recover the exact same ranking among methods as in Table 1

Following recent works in ensembling [9, 50, 64], we have mainly focused on the $\mathrm{NLL}_{c}$ metric for in-domain test set. Indeed, [2] have shown that "comparison of [...] ensembling methods without temperature scaling (TS) [25] might not provide a fair ranking". Nevertheless in Table 8 , we found that Negative Log-Likelihood (NLL) (without TS) leads to similar conclusions as $\mathrm{NLL}_{c}$ (after TS).

The TS even mostly seems to benefit to poorly calibrated models, as shown by the calibration criteria $E x$ pected Calibration Error (ECE, $\downarrow, 15$ bins). ECE measures how confidences match accuracies. MixMo attenuates over-confidence in large networks and thus reduces ECE. In our case, combining ensembling and data augmentation improves calibration [79]. Note that the appropriate measure of calibration is still under debate [56]. Notably, [2] have also stated that, despite being widely used, ECE is biased and unreliable: we can confirm that we found ECE to be dependant to hyper-parameters and implementation details. Due to space constraints and these pitfalls, we have not included this controversial metric in the main paper.

\subsection{Training dynamics}

Fig. 12 showcases training dynamics for probability $p \in$ $[0,1]$ of patch mixing (see Section 4.3.2). In the remaining $1-p$, we interpolate features linearly. For $p=0$, we recover our Linear-MixMo; for $p=0.5$, we recover our CutMixMo. In all approaches, $p$ is linearly reduced towards 0 beyond the $\frac{11}{12}$ of the training epochs, i.e. from epoch 275 to 300 on CIFAR. As we sum at inference, this reduces the train-test distribution gap and slightly increases individual accuracy during the final epochs (lower left in Fig. 12,

Diversity is measured by the ratio-error, the ratio between the number of samples on which only one of the two predictor is wrong, divided by the number of samples on which they are both wrong. It is positively correlated with $p$. However, individual accuracies first increase with $p$ until $p=0.6$, then the tendency is reversed. Overall, best ensemble performances in terms of accuracy (Top1) and uncertainty (NLL) estimation are obtained with $p \in[0.5,0.6]$. Most importantly, we note that the performance gaps are consistent and stable along training. 


\subsection{Mixed sample data augmentations}

We have drawn inspiration from MSDA techniques to design our mixing block $\mathcal{M}$. In particular, Section 4.3.2 compared different $\mathcal{M}$ based on recent papers. Fig. 13 provides the reader a visual understanding of their behaviour, which we explain below.

MixUp [86] linearly interpolates between pixels values: $m_{x}\left(x_{i}, x_{k}, \lambda\right)=\lambda x_{i}+(1-\lambda) x_{k}$. The remaining methods fall under the label of binary MSDA: $m_{x}\left(x_{i}, x_{k}, \lambda\right)=$ $\mathbb{1}_{m} \odot x_{i}+\left(\mathbb{1}-\mathbb{1}_{m}\right) \odot x_{k}$ with $\mathbb{1}_{m}$ a mask with binary values $\{0,1\}$ and area of ratio $\lambda$. They diverge in how this mask is created. The horizontal concatenation, also found in [68], simply draws a vertical line such that every pixel to the left belongs to one sample and every pixel to the right belongs to the other. Similarly, we define a vertical concatenation with an horizontal line. PatchUp [17] adapted DropBlock [24]: a canvas $C$ of patches is created by sampling for every spatial coordinate from the Bernoulli distribution $\operatorname{Ber}\left(\lambda^{\prime}\right)$ (where $\lambda^{\prime}$ is a recalibrated value of $\lambda$ ): if the drawn binary value is 1 , a patch around that coordinate is set to 1 on the final binary mask $\mathbb{1}_{m}$. PatchUp was designed for in-manifold mixing with a different mask by channels. However, duplicating the same 2D mask in all channels for $\mathcal{M}$ performs better in our experiments. FMix [29] selects a large contiguous region in one image and pastes it onto another. The binary mask is made of the top- $\lambda$ percentile of pixels from a low-pass filtered 2D map $G$ drawn from an isotropic Gaussian distribution. CowMix [20, 21] selects a cow-spotted set of regions, and is somehow similar to FMix with a Gaussian filtered 2D map $G$. CutMix [83] was inspired by CutOut [13]. Formally, we sample a square with edges of length $R \sqrt{\lambda}$, where $R$ is the length of an image edge. Note that this sometimes leads to non square rectangles when the initially sampled square overlaps with the edge from the original image. We adjust our $\lambda$ a posteriori
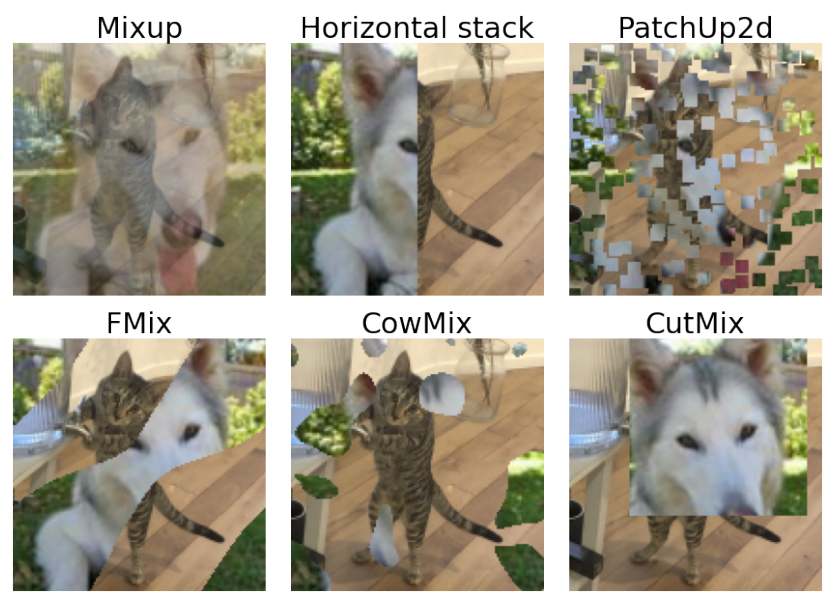

Figure 13: Common MSDA procedures with $\lambda=0.5$. to fix this boundary effect. Regarding the hyper-parameters, we use in $\mathcal{M}$ those provided in the seminal papers, except for sampling of $\kappa$ where we set $\alpha=2$ in all setups.

Note we consider both versions of MixUp (in-pixel and manifold) in this paper, but only the in-pixel version of CutMix. Indeed, the manifold version of CutMix was shown in the seminal CutMix paper [83] to be inferior to the standard in-pixel variant.

\subsection{Hyper-parameter $\alpha$}

In Fig. 14. we study the impact of different values of $\alpha$, parameterizing the sampling law for $\kappa \sim \operatorname{Beta}(\alpha, \alpha)$. For high values of $\alpha$, the interval of $\kappa$ narrows down around 0.5. Diversity is therefore decreased: we speculate this is because we do not benefit anymore from lopsided updates. The opposite extreme, when $\alpha=1$, is equivalent to uniform distribution between 0 and 1 . Therefore diversity is increased, at the cost of lower individual accuracy due to less stable training. For simplicity, we set $\alpha=2$. ManifoldMixup [76] selected the same value on CIFAR-100. However, this value could be fine tuned on the target task: e.g. in Fig. 14, $\alpha=4$ seems to perform best for Cut-MixMo on CIFAR-100 with WRN-28-10 with $r=3, p=0.5$ and $b=2$.

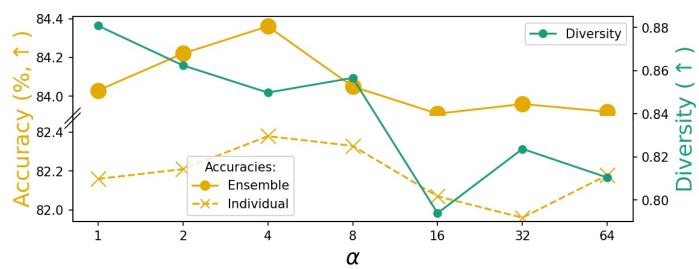

Figure 14: Diversity/accuracy as function of $\alpha$.

\subsection{Preliminary ImageNet experiments}

To further prove MixMo's ability to scale to more complex problems, we also conduct a preliminary study of its behavior on the larger scale ImageNet dataset [12]. Following the protocol outlined in the seminal MIMO paper [30], we consider variations on the standard ResNet-18 in the form of ResNet-18-w networks where $w$ is multiplicative width factor.

These first experiments confirm that MixMo performs well when networks are overparameterized. For values of $w \geq 5$, our network at the end of training outperforms both Vanilla and CutMix baselines. For example, with a ResNet-18-5 backbone, Cut-MixMo (78.20\% Top1, 0.867 NLLc) improves over Vanilla $(76.47 \%, 1.121)$ and CutMix $(77.40 \%, 1.263)$. This remains the case for a ResNet-18-7 backbone with Cut-MixMo (78.55\% Top1, 0.846 NLLc) outperforming Vanilla $(76.86 \%, 1.100)$ and CutMix $(77.18 \%, 1.190)$. 
Table 9: Summary: WRN-28- $w$ on CIFAR-100. $b=4$.

\begin{tabular}{|c|c|c|c|c|c|c|c|c|c|c|c|}
\hline \multirow{2}{*}{$\begin{array}{l}\text { Width } \\
w\end{array}$} & \multirow{2}{*}{$\begin{array}{c}\text { Approach } \\
\text { CutMix }\end{array}$} & \multicolumn{2}{|c|}{$1-\mathrm{Net}$} & \multicolumn{2}{|c|}{ 2-Nets } & \multicolumn{2}{|c|}{ Linear-MixMo } & \multicolumn{2}{|c|}{ Cut-MixMo } & \multicolumn{2}{|c|}{ 2-Cut-MixMos } \\
\hline & & - & $\checkmark$ & - & $\checkmark$ & - & $\checkmark$ & - & $\checkmark$ & - & $\checkmark$ \\
\hline \multirow{3}{*}{2} & \multirow{3}{*}{$\begin{array}{c}\text { Top1 } \\
\text { NLL }_{c} \\
\text { \# params }\end{array}$} & 6.44 & 78.06 & 79.16 & 80.81 & 75.82 & 76.36 & 75.66 & 75.17 & 76.98 & 76.11 \\
\hline & & 0.921 & 0.815 & 0.776 & 0.695 & 0.841 & 0.824 & 0.824 & 0.846 & 0.7661 & 0.798 \\
\hline & & \multicolumn{2}{|c|}{$1.48 \mathrm{M}$} & \multicolumn{2}{|c|}{$2.95 \mathrm{M}$} & \multicolumn{4}{|c|}{$1.49 \mathrm{M}$} & \multicolumn{2}{|c|}{$2.99 \mathrm{M}$} \\
\hline \multirow{3}{*}{3} & \multirow{3}{*}{$\begin{array}{c}\text { Top1 } \\
\text { NLL }_{c} \\
\text { \# params }\end{array}$} & 77.95 & 80.70 & 80.85 & 83.14 & 78.51 & 80.74 & 79.81 & 79.85 & 80.78 & 81.20 \\
\hline & & 0.862 & 0.750 & 0.738 & 0.644 & 0.760 & 0.696 & 0.693 & 0.702 & 0.635 & 0.650 \\
\hline & & \multicolumn{2}{|c|}{$3.31 \mathrm{M}$} & \multicolumn{2}{|c|}{$6.62 \mathrm{M}$} & \multicolumn{4}{|c|}{$3.33 \mathrm{M}$} & \multicolumn{2}{|c|}{$6.66 \mathrm{M}$} \\
\hline \multirow{3}{*}{4} & \multirow{3}{*}{$\begin{array}{c}\text { Top1 } \\
\text { NLL }_{c} \\
\text { \# params }\end{array}$} & 78.84 & 81.55 & 81.48 & 83.93 & 80.4 & 81.66 & 81.68 & 81.69 & 82.57 & 82.58 \\
\hline & & 0.824 & 0.711 & 0.711 & 0.609 & 0.712 & 0.656 & 0.646 & 0.635 & 0.590 & 0.588 \\
\hline & & \multicolumn{2}{|c|}{$5.87 \mathrm{M}$} & \multicolumn{2}{|c|}{$11.74 \mathrm{M}$} & \multicolumn{4}{|c|}{$5.89 \mathrm{M}$} & \multicolumn{2}{|c|}{$11.79 \mathrm{M}$} \\
\hline \multirow{3}{*}{5} & \multirow{3}{*}{$\begin{array}{c}\text { Top1 } \\
\text { NLL }_{c} \\
\text { \# params }\end{array}$} & 79.75 & 82.55 & 82.18 & 84.60 & 80.95 & 83.06 & 83.11 & 83.34 & 83.97 & 84.31 \\
\hline & & 0.813 & 0.686 & 0.693 & 0.596 & 0.703 & 0.617 & 0.598 & 0.591 & 0.549 & 0.546 \\
\hline & & \multicolumn{2}{|c|}{$9.16 \mathrm{M}$} & \multicolumn{2}{|c|}{$18.32 \mathrm{M}$} & \multicolumn{4}{|c|}{$9.19 \mathrm{M}$} & \multicolumn{2}{|c|}{$18.39 \mathrm{M}$} \\
\hline \multirow{3}{*}{7} & Top1 & 81.14 & 83.71 & 82.94 & 85.52 & 82.4 & 84.51 & 84.32 & 84.94 & 85.50 & 85.90 \\
\hline & $\mathrm{NLL}_{c}$ & 0.764 & 0.648 & 0.673 & 0.573 & 0.675 & 0.581 & 0.562 & 0.543 & 0.516 & 0.498 \\
\hline & \# params & & $2 \mathrm{M}$ & & & & & & & 35.9 & \\
\hline & Top1 & 81.63 & 84.05 & 83.17 & 85.74 & 83.08 & 85.47 & 85.40 & 85.77 & 86.04 & 86.63 \\
\hline 10 & $\mathrm{NLL}_{c}$ & 0.750 & 0.644 & 0.668 & 0.571 & 0.656 & 0.558 & 0.535 & 0.524 & 0.494 & 0.479 \\
\hline & \# params & & & & & & & & & & \\
\hline & Top1 & 82.01 & 84.31 & 83.47 & 85.80 & 83.7 & 86.05 & 85.76 & 86.19 & 86.58 & 87.11 \\
\hline 14 & $\mathrm{NLL}_{c}$ & 0.730 & 0.645 & 0.656 & 0.569 & 0.648 & 0.545 & 0.527 & 0.518 & 0.488 & 0.473 \\
\hline & \# params & 71. & $5 \mathrm{M}$ & & & & & & & & $8 \mathrm{M}$ \\
\hline
\end{tabular}

\subsection{Ensemble of Cut-MixMo with CutMix}

Fig. 15 plots performance for different widths $w$ in WRN-28- $w$ and varying number of ensembled networks $N$ : two vertically aligned points have the same parameter budget. Indeed, the total number of parameters in our

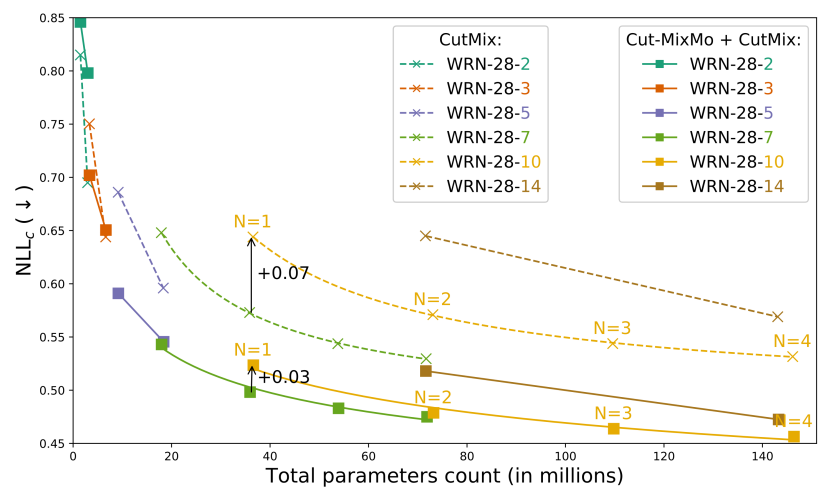

Figure 15: Ensemble effectiveness $\left(\mathrm{NLL}_{c} / \#\right.$ params). We slide the width in WRN-28- $w$ and numbers of members $N$. CutMix data augmentation. Interpolations through power laws [50] when more than 2 points are available. architectures has been used as a proxy for model complexity, as in [9, 50]. The increase in the total number of weights in MixMo is visually almost unnoticeable. Precisely, with WRN-28-10, MixMo $(M=2)$ has $36.60 \mathrm{M}$ weights vs. $36.53 \mathrm{M}$ standardly $(+0.2 \%)$. Moreover, the number of flops is 5.9571G Flops for MixMo vs. $5.9565 \mathrm{G}$ Flops standardly $(+0.01 \%)$. That's why we state we achieve ensembling (almost) "for free".

We compare ensembling with CutMix rather than standard pixels data augmentation, as previously done in Fig.6 from Section 4.6. CutMix induces additional regularization and label smoothing: empirically, it improves all our approaches. For a fixed memory budget, a single network usually performs worse than an ensemble of several mediumsize networks: we recover the Memory Split Advantage even with CutMix. However, Cut-MixMo challenges this by remaining closer to the lower envelope. In other words, parameters allocation (more networks or bigger networks) has less impact on results. This is due to Cut-MixMo's ability to better use large networks.

In Table 9, we summarize several experiments on CIFAR-100. Among other things, we can observe that large vanilla networks tend to gain less from ensembling [50]: e.g. 2 vanillas WRN-28-10 (83.17\% Top1, $\left.0.668 \mathrm{NLL}_{c}\right)$ 
do not perform much better than 2 WRN-28-7 (82.94\%, 0.673). This remains true even with CutMix: $(85.74 \%$, $0.571)$ vs. $(85.52 \%, 0.573)$. We speculate this is related to wide networks' tendency to converge to less diverse solutions, as studied in [55]. Contrarily, MixMo improves the ensembling of large networks, with $(86.04 \%, 0.494)$ vs. $(85.50 \%, 0.517)$ on the same setup. When additionally combined with CutMix, we obtain state of the art $(86.63 \%$, $0.479)$ vs. $(85.90 \%, 0.498)$. This demonstrates the importance of Cut-MixMo in cooperation with standard pixels data augmentation. It attenuates the drawbacks from overparameterization This is of great importance for practical efficiency: it modifies the optimal network width for realworld applications.

\subsection{Pseudo Code}

Finally, the pseudocode in Algorithm 11 describes the procedure behind Cut-MixMo with $M=2$. 


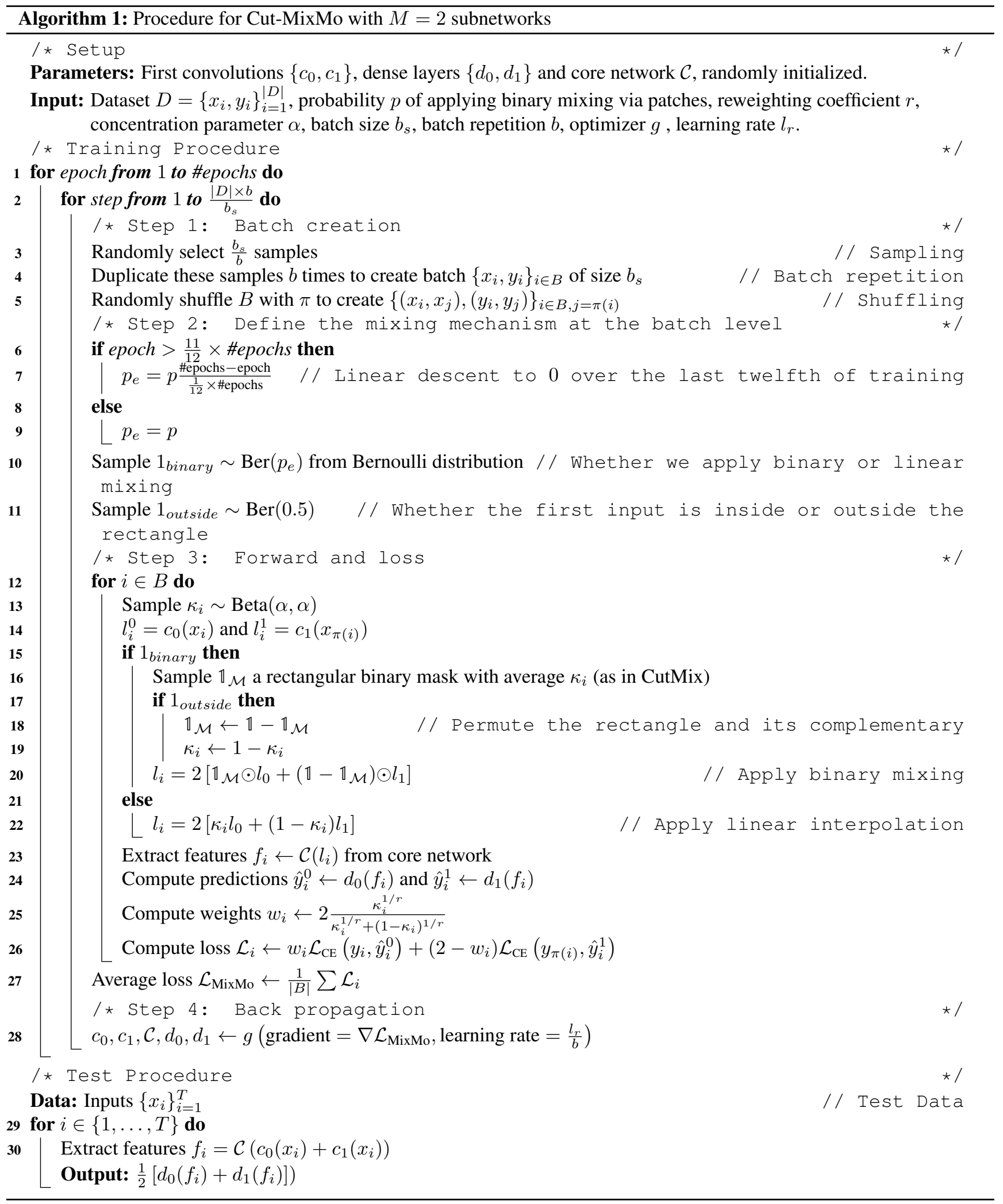

